\title{
HARMONIC INTEGRALS ON ALMOST PRODUCT MANIFOLDS
}

\author{
BY \\ BRUCE L. REINHART(1)
}

An almost product manifold is one on which the tangent bundle has a differentiable decomposition as the direct sum of two subbundles. We assume that each of the subspaces thus defined in the tangent space is integrable; then local coordinates $\left(x^{1}, \cdots, x^{p}, y^{1}, \cdots, y^{q}\right)$ can be found in some neighborhood $U$ of any point such that $\left\{\partial / \partial x^{i}\right\}$ generates the one subspace over $U$ and $\left\{\partial / \partial y^{j}\right\}$ generates the other. Operators $d^{\prime}, \delta^{\prime}$, and $\Delta^{\prime}=d^{\prime} \delta^{\prime}+\delta^{\prime} d^{\prime}$ are defined on differential forms, all of them differentiating with respect to $x$ alone. If

$$
\begin{gathered}
\phi=\sum_{i_{1}<\cdots<i_{r}, j_{1}<\cdots<j_{s}} \phi_{i_{1} \cdots i_{r} j_{1} \cdots j_{s}} d x^{i_{1}} \wedge \cdots \wedge d x^{i_{r}} \wedge d y^{j_{1}} \wedge \cdots \wedge d y^{j_{s}}, \\
\left(d^{\prime} \phi\right)_{i_{1} \cdots i_{r}+j_{1} \cdots j_{s}}=\sum_{k, k_{1}<\cdots<k_{r}} \delta_{i_{1} \cdots i_{r}}^{k k_{1} \cdots k_{r}} \frac{\partial \phi_{k_{1}} \cdots k_{r} j_{1} \cdots j_{s}}{\partial x^{k}}
\end{gathered}
$$

and

$$
\left(\Delta^{\prime} \phi\right)_{i_{1} \cdots i_{r} j_{1} \cdots j_{s}}=-g^{\prime i j} \frac{\partial^{2} \phi_{i_{1} \cdots i_{r} j_{1} \cdots j_{s}}}{\partial x^{i} \partial x^{j}}+\text { lower order terms. }
$$

Here we have assumed that the metric is of the form $d s^{2}=\sum_{i, j=1}^{p} g_{i j}^{\prime}(x, y) d x^{i} d x^{j}$ $+\sum_{i, j=1}^{q} g_{i j}^{\prime \prime}(x, y) d y^{i} d y^{j}$.

The operator $d^{\prime}$ satisfies $d^{\prime 2}=0$, hence defines a cohomology theory of differential forms. It is natural to ask whether a Hodge theorem for this cohomology exists; that is, whether each cohomology class contains one and only one form $\phi$ satisfying $\Delta^{\prime} \phi=0$. Since $\Delta^{\prime}$ is a semi-definite operator, some restriction is necessary. Our principal result is that a theorem of the desired type holds on a compact manifold under appropriate restrictions on the metric and the class of forms admitted. The proof of the theorem uses construction of a Green's operator $G^{\prime}$ satisfying $G^{\prime} d^{\prime} \phi=d^{\prime} G^{\prime} \phi$ and $G^{\prime} \Delta^{\prime} \phi=\Delta^{\prime} G^{\prime} \phi=\phi-H^{\prime} \phi$, where $H^{\prime}$ is the projection on the kernel of $\Delta^{\prime}$. In general, $G^{\prime}$ is not completely continuous and the kernel of $\Delta^{\prime}$ is infinite dimensional.

I wish to express my gratitude to Professor D. C. Spencer, who was always ready to discuss my problems with me. His suggestions on many points in the

Presented to the Society April 21, 1956; received by the editors April 4, 1956 and, in revised form, November 23, 1956.

(1) This paper was prepared while the author was partially supported by a research project at Princeton University sponsored by the Office of Ordnance Research, U. S. Army. It is based on a substantial portion of the author's thesis. 
writing of this paper are deeply appreciated. A word of thanks is also due to Professor S. S. Chern, who made several useful suggestions.

1. Preliminary remarks. Let $V$ be a connected differentiable manifold of dimension $n$ and class $C^{\infty}$. Denote its tangent bundle by $T(V)$, the bundle of tangent $p$-vectors by $\Lambda^{p} T(V)$ or $T^{p}(V)$, and the bundle of Grassmann algebras by $\Lambda T(V)=\sum_{p=0}^{n} T^{p}(V)$. Here $\Lambda^{0} T(V)$ is the trivial bundle of real numbers, and $\Lambda^{1} T(V)=T(V)$. Let $\Phi(V)$ be the bundle of cotangents, with other notations, $\Lambda^{p} \Phi(V), \Lambda \Phi(V)$, etc., analogous to those for $T(V)$. Then a section of $\Lambda \Phi(V)$ is a differential polynomial in the usual sense. Let $d$ denote the operation of exterior differentiation on such a polynomial or on $\Lambda \Phi(V)$.

The manifold $V$ has an almost product structure $(P, Q)$ if there exist projection homomorphisms $P, Q: T(V) \rightarrow T(V)$ such that:

(i) If $T_{b}(V)$ is the tangent space to $V$ at the point $b$ of $V$, then $P\left(T_{b}(V)\right)$ $\subset T_{b}(V)$ and $Q\left(T_{b}(V)\right) \subset T_{b}(V)$.

(ii) $T_{b}(V)=P\left(T_{b}(V)\right) \oplus Q\left(T_{b}(V)\right)$, the sum being direct.

(iii) $P$ and $Q$ are $C^{\infty}$ maps.

Let $p$ (resp. $q$ ) denote the dimension of $P T_{b}(V)$ (resp. $Q T_{b}(V)$ ); $p$ is independent of the point $b$. The almost product structure induces projection operators $\coprod_{r, s *}$ in $\Lambda T(V)$ and $\prod_{r, s}$ in $\Lambda \Phi(V)$. A vector field which is a section of $\prod_{r, s *} \Lambda T(V)=T^{r, s}(V)$ is said to be of type $(r, s)$, and similarly for $\Lambda \Phi(V)$. If $V$ and $\bar{V}$ are manifolds with almost product structures $(P, Q)$ and $(\bar{P}, \bar{Q})$, then a map $f: V \rightarrow \bar{V}$ is said to be of type $(m, n)$ with respect to these structures if $\prod_{r+m, s+n *} \circ f_{*}=f_{*} \circ \prod_{r, s *}$. Here $f_{*}: \Lambda T(V) \rightarrow \Lambda T(\bar{V})$ is the map induced by $f$.

An operator $h$ on $\Lambda T(V)$ or $\Lambda \Phi(V)$ may be decomposed into various types according to the definition $\prod_{m, n} h=\sum_{r, s} \prod_{r+m, s+n} \circ h \circ \prod_{r, s}$. If the operator $d$ of exterior differentiation is so decomposed, we find that $d=d_{2}^{\prime}+d_{1}^{\prime}$ $+d_{1}^{\prime \prime}+d_{2}^{\prime \prime}$, where the components are of types $(2,-1),(1,0),(0,1)$, and $(-1,2)$ respectively. For a proof of this decomposition, see Guggenheim and Spencer [10]. We may also define an operator $d^{\prime}: \Phi^{p}(V) \rightarrow \Phi^{p+1}(V)$ by the axioms:

(i) If $\phi$ is in $\Lambda^{0} \Phi_{b}(V)$ and $v$ is in $T_{b}(V)$ where $b$ is a point of $V$, then $\left(d^{\prime} \phi\right)(v)=(P v)(\phi)$.

(ii) If $\phi$ is in $\Lambda^{0} \Phi_{b}(V)$, then $\left(d^{\prime} d+d d^{\prime}\right) \phi=0$.

(iii) If $\phi$ is in $\Lambda^{p} \Phi_{b}(V)$ and $\psi$ is in $\Lambda \Phi_{b}(V)$, then $d^{\prime}(\phi \wedge \psi)=d^{\prime} \phi \wedge \psi+(-1)^{p} \phi$ $\wedge d^{\prime} \psi$.

Then $d^{\prime}=2 d_{2}^{\prime}+d_{1}^{\prime}-d_{2}^{\prime \prime}$ (Guggenheim and Spencer [10]). Analogously, we define $d^{\prime \prime}$ and have $d^{\prime \prime}=2 d_{2}^{\prime \prime}+d_{1}^{\prime \prime}-d_{2}^{\prime}$. Adding these relations, we get $d=d^{\prime}+d^{\prime \prime}$.

The operators $d^{\prime}$ and $d^{\prime \prime}$ are not in general differentials; that is $\left(d^{\prime}\right)^{2} \neq 0$. However, they are differentials if and only if the structure is integrable, by which we mean that the Poisson bracket of two vector fields of type $P$ (resp. $Q$ ) is again a vector field of the same type. In fact, $\left(d^{\prime}\right)^{2}=0$ if and only 
if $d^{\prime}=d_{1}^{\prime}$ and $d^{\prime \prime}=d_{1}^{\prime \prime}$, which is in turn equivalent to the integrability (Guggenheim and Spencer [10]).

The structure being integrable, we can apply the Frobenius theorem to obtain local coordinates $\left(x^{1}, \cdots, x^{p}, y^{1}, \cdots, y^{q}\right)$ in the neighborhood of any point such that $P T(V)$ is spanned by $\left\{\partial / \partial x^{i}\right\}$ and $Q T(V)$ by $\left\{\partial / \partial y^{j}\right\}$. In fact, suppose that in the neighborhood $U$, the almost product structure is given by differential forms $\Omega=\omega^{1} \wedge \cdots \wedge \omega^{p}$ and $\Theta=\theta^{1} \wedge \cdots \wedge \theta^{q}$; this is possible because any subspace of the cotangent space is representable locally by a nonzero decomposable form. Then $\Omega \wedge \Theta \neq 0$. Because the structure is integrable, we have that $d \Omega$ is in the ideal generated by $\Omega$, while $d \Theta$ is in the ideal generated by $\Theta$. By Frobenius theorem, there exist local coordinates $\left(x^{1}, \cdots, x^{n}\right)$ such that $\Omega=\lambda d x^{1} \wedge \cdots \wedge d x^{p}$, and local coordinates $\left(y^{1}, \cdots, y^{n}\right)$ such that $\Theta=\mu d y^{1} \wedge \cdots \wedge d y^{q}$, where $\lambda$ and $\mu$ are nonzero functions. (A proof may be found in Chern [3], for example.) But $d x^{1} \wedge \cdots \wedge d x^{p}$ $\wedge d y^{1} \wedge \cdots \wedge d y^{q}=1 / \lambda \mu \Omega \wedge \Theta \neq 0$; hence $\left(x^{1}, \cdots, x^{p}, y^{1}, \cdots, y^{q}\right)$ form a coordinate system, which clearly has the desired properties.

In terms of such a coordinate system, a differential form of type $(r, s)$ may be written as

$$
\phi=\sum_{(i)(j)} \phi_{i_{1}} \cdots i_{r} j_{1} \cdots j_{s} d x^{i_{1}} \wedge \cdots \wedge d x^{i_{r}} \wedge d y^{j_{1}} \wedge \cdots \wedge d y^{j_{s}}
$$

where (i) means an ordered set of indices $i_{1}<\cdots<i_{r}$. An index $i_{\alpha}$ (resp. $j_{\beta}$ ) will be said to be of the first (resp. second) type; we shall use the convention that an index of the first type is written before one of second type.

The notion of integrable almost product structure is related to that of laminated manifold (variété feuilletée) discussed by Reeb [18]. On such a manifold one has given a single integrable subspace, rather than a complementary pair.

In the case of the operator $d$, there is a theorem that for forms of positive degree, $d \phi=0$ implies that locally $\phi=d \psi$. This theorem, called the Poincaré lemma, is also true for $d^{\prime}$ and forms of type $(r, s)$ with $r>0$. In fact, if $F$ is a homotopy of the kind described below, then $F$ induces a chain homotopy $k^{\prime}$ such that $d^{\prime} k^{\prime} \phi+k^{\prime} d^{\prime} \phi=\phi$ for all forms $\phi$ of the proper type. For a proof of this statement, see Guggenheim and Spencer [10]. In order to construct the homotopy $F$, it is sufficient to assume the integrability of the almost product structure; then $F$ deforms $\left(x^{1}, \cdots, x^{p}, y^{1}, \cdots, y^{q}\right)$ into $(0, \cdots, 0$, $\left.y^{1}, \cdots, y^{q}\right)$ in the obvious manner. A similar theory may be constructed for $d^{\prime \prime}$.

We may introduce on the manifold $V$ a Riemannian metric such that the subspaces $P T(V)$ and $Q T(V)$ are orthogonal. In fact, $\Phi(V)$ is a bundle having as structure group $G L(p, R) \oplus G L(q, R)$ where $p$ is the dimension of $P \Phi(V)$ at a point and $G L(p, R)$ is the general linear group with real coefficients. Dividing out by the subgroup $O(p, R) \oplus O(q, R)$ of direct sums of orthogonal matrices, we get the direct sum $H(p, R) \oplus H(q, R)$ of sets of posi- 
tive definite symmetric matrices. Consider the bundle with this fibre weakly associated to the bundle of cotangents. The fibre is topologically the product of a cell with a cell, hence is also a cell. Therefore, the bundle has a crosssection, and the group of the cotangent bundle is reducible to $O(p, R)$ $\oplus O(q, R)$. The cross-section defines a Riemannian metric which has the desired properties. If the structure is integrable and local product coordinates are used, this metric will have the form $d s^{2}=g_{i j}^{\prime} d x^{i} d x^{i}+g_{i j}^{\prime \prime} d y^{i} d y^{i}$, where the coefficients are functions of both $x$ and $y$ in general. For further details on the preceding construction, see Steenrod [27, p. 58] where an ordinary Riemann metric is constructed for a differentiable manifold.

Henceforth, let us assume an almost product metric given on $V$. In this case, we may define an operator $\delta^{\prime}=(-1)^{n m+n+1} * d^{\prime} *$, where $\delta^{\prime}$ operates on a form of total degree $m$ and $n$ is the dimension of $V$. Here $*$ is the duality operator defined by the metric. Similarly define $\delta^{\prime \prime}$. Since $\delta=(-1)^{n m+n+1} * d *$, we see at once that $\delta=\delta^{\prime}+\delta^{\prime \prime}$. We also construct special Laplacian operators by defining $\Delta^{\prime}=d^{\prime} \delta^{\prime}+\delta^{\prime} d^{\prime}, \Delta^{\prime \prime}=d^{\prime \prime} \delta^{\prime \prime}+\delta^{\prime \prime} d^{\prime \prime}$, and $\widetilde{\Delta}=\Delta^{\prime}+\Delta^{\prime \prime}$. For a general discussion of the definitions of $*, \delta, \Delta$, and the inner product of forms on a manifold, see de Rham [19, pp. 119-132].

Since we are dealing with an almost product metric, we have $* \prod_{r, s}$ $=\prod_{p-r, q-s} *$. Thus $\delta^{\prime}$ is of type $(-1,0)$ for an integrable structure, while $\Delta^{\prime}, \Delta^{\prime \prime}$, and $\tilde{\Delta}$ preserve types. In what follows we assume the manifold to be compact. Defining the inner product $(\phi, \psi)$ in the usual way, we have $(d \phi, \psi)$ $=(\phi, \delta \psi)$ and $\left(\prod_{r, s} \phi, \prod_{t, u} \psi\right)=0$ unless $r=t$ and $s=u$. Making use of these facts, we see that $\left(d^{\prime} \phi, \psi\right)=\left(\phi, \delta^{\prime} \psi\right)$ and $\left(d^{\prime \prime} \phi, \psi\right)=\left(\phi, \delta^{\prime \prime} \psi\right)$. These in turn imply that $\left(\Delta^{\prime} \phi, \psi\right)=\left(\phi, \Delta^{\prime} \psi\right),\left(\Delta^{\prime \prime} \phi, \psi\right)=\left(\phi, \Delta^{\prime \prime} \psi\right)$, and $(\tilde{\Delta} \phi, \psi)=(\phi, \tilde{\Delta} \psi)$. Furthermore, $\Delta^{\prime} \phi=0$ if and only if $d^{\prime} \phi=\delta^{\prime} \phi=0$, for $\left(\Delta^{\prime} \phi, \phi\right)=\left(d^{\prime} \phi, d^{\prime} \phi\right)$ $+\left(\delta^{\prime} \phi, \delta^{\prime} \phi\right)$.

An almost product metric will be said to be torsionless if the parallel transport by means of the metric of a vector $v$ in $P T(V)$ (resp. $Q T(V)$ ) is also a vector in $P T(V)$ (resp. $Q T(V)$ ). This condition may be interpreted as saying that the holonomy group is reducible $\left({ }^{2}\right)$. Writing out the equation for parallel transport and replacing the Christoffel symbols by their expressions in terms of the metric tensor and its derivatives, we see that the metric is torsionless if and only if $\partial g_{i j}^{\prime} / \partial y^{k}=\partial g_{i j}^{\prime \prime} / \partial x^{k}=0$. In other words, $d s^{2}=g_{i j}^{\prime}(x) d x^{i} d x^{j}$ $+g_{i j}^{\prime \prime}(y) d y^{i} d y^{j}$. Referring to the expression for the components of the induced connection, of the Riemann curvature, and of the Ricci curvature in terms of the components of the metric tensor, it is immediate that each of these splits in a manner exactly analogous to the splitting of the metric. A theorem of de Rham states that a complete, simply connected Riemann manifold with an integrable almost product structure and a torsionless metric is a product space; see de Rham [20].

(2) The holonomy group was defined and studied locally by E. Cartan [2]. Manifolds with reducible holonomy groups are studied by Borel and Lichnerowicz [1] and de Rham [20]. 
In developing the potential theory of $\Delta^{\prime}$, it is convenient to use the language of sheaves. All that is required in this paper is the fundamental definitions, which we shall now give. No deep sheaf-theoretic theorems will be needed.

A sheaf (over the real numbers) is a triple $(S, \pi, X)$ where $S$ and $X$ are topological spaces and $\pi$ is a mapping called the projection of $S$ onto $X$, such that:

(i) $S_{x}=\pi^{-1}(x)$ is a real vector space for each $x$ in $X$. ( $S_{x}$ is called the stalk over $x$.)

(ii) $\pi$ is a local homeomorphism.

(iii) The operations of addition and of multiplication by a real number are continuous.

A section of $S$ over an open set $U$ of $X$ is a continuous mapping $f: U \rightarrow S$ such that $\pi \circ f(x)=x$ for all $x$ in $U$. The set of all sections of $S$ over $U$ is denoted by $\Gamma(S, U)$.

A sheaf may also be defined by giving sets of sections. Let $\mathfrak{U}=\left\{U_{\alpha}\right\}$ be a base for the open sets of a space $X$. Let $S_{\alpha}$ (given a priori) be the vector space of sections over $U_{\alpha}$. If $U_{\alpha}$ contains $U_{\beta}$, let there be given a map $r_{\alpha \beta}: S_{\alpha} \rightarrow S_{\beta}$. The sheaf $S$ is defined as the direct limit of this system of vector spaces and maps. In other words, a point $s$ in $S_{x}$ is an equivalence class under the equivalence relation: $s_{\alpha} \sim s_{\beta}$ if and only if there exists a $U_{\gamma}$ in $\mathfrak{U}$ such that $r_{\alpha \gamma} s_{\alpha}=r_{\beta \gamma} s_{\beta}$. Here $s_{\alpha}$ is in $S_{\alpha}, s_{\beta}$ is in $S_{\beta}$, and $x$ is in $U_{\gamma}$. A sheaf constructed in this way is called a sheaf of germs; there exist many such of importance.

The definition here given for a sheaf is due to Lazard, who worked it out while attending the Paris seminar of $\mathrm{H}$. Cartan. For more information on sheaves and for examples, see the Paris notes [23] or the paper of Serre [24, Chapter I]. A detailed discussion of much of the material in this $\$ 1$ will be found in the forthcoming monograph of Nickerson and Spencer [17].

2. Summary. We now state briefly the results which will be proved in this paper. First, we consider in $\S \S 3$ and 4 examples of almost product structures on the 3-sphere and on the torus. Another example is a connection in a principal fibre bundle. For the particular case of the torus, a classification of almost product structures is given; for the simpler ones, the properties of $\Delta^{\prime}$ are also analyzed by a method of Kodaira. It is shown that the inverse (Green's operator) for $\Delta^{\prime}$ need not exist, even for $C^{\infty}$ forms orthogonal to the solutions of $\Delta^{\prime} \phi=0$. Also, the $d^{\prime}$ cohomology of forms is not isomorphic to the space of solutions; in other words, the analogue of Hodge's theorem is false. These counter-examples are structures with torsionless metrics; they show that even in this case considerable difficulty is to be expected in the harmonic theory.

The operator $\tilde{\Delta}$ is the subject of $\S 5$. The solutions of $\tilde{\Delta} \phi=0$ are precisely those forms of pure type which are harmonic in the usual sense. $\Delta=\tilde{\Delta}$ if and only if $\Delta$ commutes with type projection operators. $\tilde{\Delta}$ is an elliptic operator; hence we can find a Green's operator by the usual methods. However, 
this operator fails to commute with $d^{\prime}, \delta^{\prime}$, and the other related operators.

In $\S 6$, the problem of constructing a Green's operator for $\Delta^{\prime}$ is taken up. We define a sheaf $E=\sum_{r, s} D_{x}^{r} \wedge D_{y}^{s}$, where $D_{x}^{r}$ is the sheaf of germs of $(r, 0)$ forms depending on $x$ alone, and analogously for $D_{\nu}^{s}$. The notion of coherent subsheaf $B$ of $E$ is defined; $E$ and all its coherent subsheaves are stable under $d^{\prime}, \delta^{\prime}$, and $\Delta^{\prime}$. Furthermore, if $H^{\prime}$ is the projection operator which maps any form into its $\Delta^{\prime}$ harmonic part, then $H^{\prime}$ maps $\Gamma(E, V)$ into $\Gamma(E, V)$ and $\Gamma(B, V)$ into $\Gamma(B, V)$. The following theorem is proved:

THEOREM. Let $V$ be a compact almost product manifold with torsionless metric. Let $\phi$ be a $C^{\infty}$ section of the sheaf $E$. Then there is a $C^{\infty}$ section $G^{\prime} \phi$ of $E$ such that $\Delta^{\prime} G^{\prime} \phi=G^{\prime} \Delta^{\prime} \phi=\phi-H^{\prime} \phi$ and $G^{\prime} H^{\prime} \phi=H^{\prime} G^{\prime} \phi=0 . G^{\prime}$ and $H^{\prime}$ are defined by choosing a coherent subsheaf $B$ of $E$ such that $\phi$ is a section of $B$, but they are independent of the choice of $B . G^{\prime}$ commutes with $d^{\prime}$ and $\delta^{\prime}$.

This theorem readily implies the isomorphism between the $d^{\prime}$ cohomology of sections of $E$ and the space of $\Delta^{\prime}$ harmonic sections of $E$. The Green's operator is constructed as the solution of a minimizing problem, following the method of Morrey and Eells [16]; the necessary differentiability lemma is proved by a modification of Kodaira's method [14]. The paragraph closes with a few comments on the Hilbert space properties of the operator $\Delta^{\prime}$. The bigrading naturally defined on the differential forms on an almost product manifold gives rise to a spectral sequence in the usual way. Another consequence of the above theorem is that in this sequence, $E_{2}$ is isomorphic to $E_{\infty}$, at least if the bigrading is taken on those forms of $C^{\infty}$ which are in the common domain of $G^{\prime}$ and $G^{\prime \prime}$ (by the theorem, this common domain includes at least the $C^{\infty}$ sections of the sheaf $E$ ).

3. General examples. Our purpose in this paragraph is to develop several examples of almost product structures on compact manifolds, in order to shed light on some of the possibilities. In the case of structures on the torus we shall examine in $\$ 4$ the potential theoretic properties; it will appear that the theory of $\Delta^{\prime}$ on a compact manifold lacks many of the results that hold for $\Delta$. In particular, the Green's operator is not everywhere defined, even for $C^{\infty}$ forms orthogonal to the harmonic space (in the sense of solutions to $\Delta^{\prime} \phi=0$ ).

Following an example of Reeb [18, pp. 112-113], we may construct an integrable almost product structure on the 3 sphere $S^{3}$. This structure is of course not group invariant, nor has it a torsionless metric. For the construction, suppose $S^{3}$ to be given by the equation $x^{2}+y^{2}+z^{2}+t^{2}=2$ in $E^{4}$. We define a one-form on $S^{3}$ by the equations

$$
\varpi= \begin{cases}d\left(x^{2}+y^{2}\right)+\left(x^{2}+y^{2}-1\right)(x d y-y d x) & \text { for } 1 \leqq x^{2}+y^{2} \leqq 2, \\ d\left(z^{2}+t^{2}\right)+\left(z^{2}+t^{2}-1\right)(z d t-t d z) & \text { for } 1 \leqq z^{2}+t^{2} \leqq 2 .\end{cases}
$$

The form $\omega$ is completely integrable, and so defines a continuous family of analytic surfaces in $S^{3}$. Define a vector field by taking the normal vectors to these surfaces in the metric induced from $E^{4}$. Any vector field has integral 
curves. Since the coefficients of $\varpi$ are of class $C^{0}$ in the neighborhood of $x^{2}+y^{2}$ $=1$, the structure is only continuous. By varying the coefficients slightly, it may be made $C^{\infty}$. A theorem recently announced by Haefliger [11] states that no analytic $n-1$ dimensional lamination of an $n$ sphere is possible.

An important example of an almost product structure is a connection in a principal fibre bundle. Such structures are not in general integrable; in fact, a necessary and sufficient condition for integrability is that the curvature of the connection be zero. The proof is not difficult, but will be omitted. It may be carried out by an analysis of the type components of $d$ and their relation to covariant differentiation and the curvature tensor, recalling that integrability is equivalent to $d^{\prime 2}=0$. This proof is due to D. C. Spencer. The theorem itself is a classical result in the case of the tangent bundle; for other bundles, a proof is given by Chern [4, pp. 54-55].

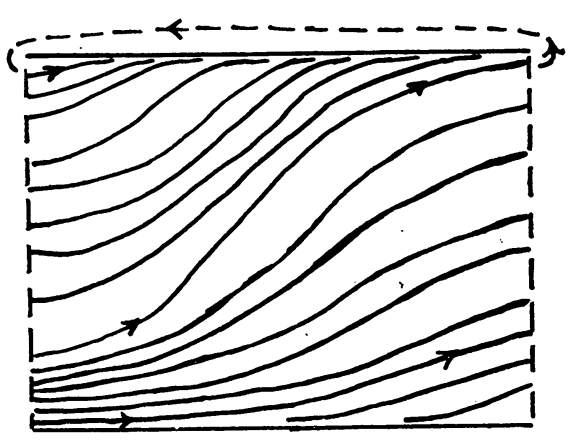

FIG. 1

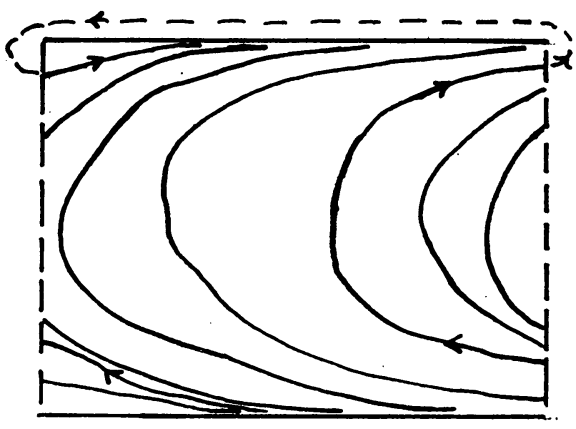

FIG. 2

Let us turn to some examples of almost product structures on compact 2 dimensional manifolds. We seek a complementary pair of nonsingular vector fields; if orientability is assumed, this means our discussion is limited to the torus. Furthermore, since vector fields are always integrable, we deal only with integrable almost product structures. Some of these structures will have torsionless metrics, but some will not.

Kneser [13] has classified topological by regular families of curves on a torus. The integral curves of a nonsingular vector field form such a family. According to this classification, if there is a closed integral curve, then the family may be thought of as consisting of a number of bands of closed curves, possibly with a countable number of bands like Figure 1 and a finite number like Figure 2 interpolated. The boundaries of each such band are closed trajectories asymptotic to each trajectory within the band. If there is no closed integral curve, then either every curve is ergodic, or else we have a highly pathological situation known as the singular case. A theorem of Denjoy [6] shows that for a nonsingular vector field of class $C^{2}$ given in the form $d y / d x=f(x, y)$ with $f(x, y)$ a doubly periodic function in the plane, the singular case cannot occur. Van Kampen [12] simplified the proof considerably, while Siegel [25] has 
shown how to extend the results to fields not given in this form.

Next we examine what happens when we suppose given an almost product structure. A few examples will prove instructive. Suppose we consider the torus given as the square $0 \leqq x<1,0 \leqq y<1$ in the plane, and consider equations with coefficients of period 1 in $x$ and $y$. Then

$$
\begin{aligned}
& \left(\sin ^{2} \pi y-1\right) d x+\left(\sin ^{2} \pi y+A\right) d y=0, \\
& \left(\sin ^{2} \pi y+A\right) d x+\left(1-\sin ^{2} \pi y\right) d y=0
\end{aligned}
$$

define an almost product structure. Figure 3 gives a picture of this structure. The dotted lines may or may not be closed curves, depending on the choiec of $A$.

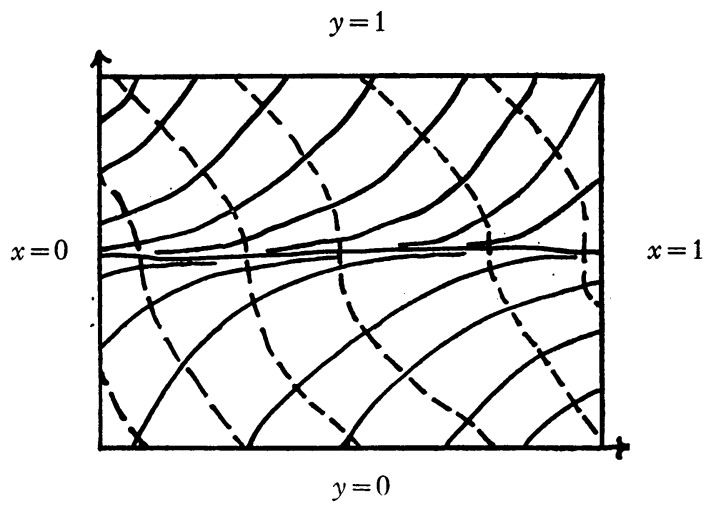

FIG. 3

$$
y=1
$$

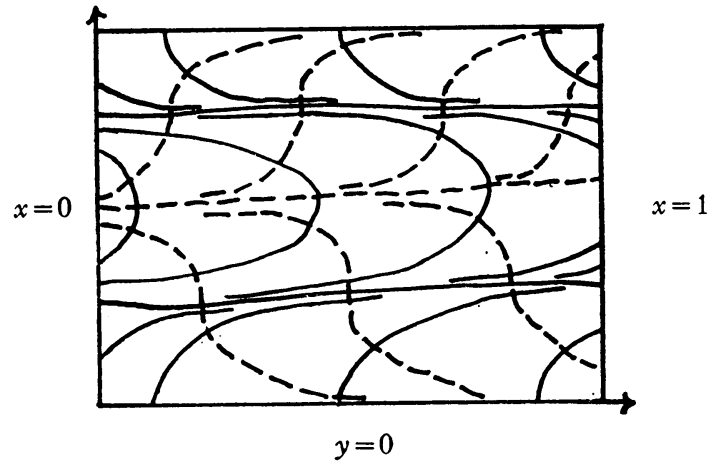

FIG. 4

With notations as in the preceding paragraph, define another structure by

$$
\begin{aligned}
& \left(1-\sin ^{2} 2 \pi y\right) d x-\sin 2 \pi y d y=0, \\
& \sin 2 \pi y d x+\left(1-\sin ^{2} 2 \pi y\right) d y=0 .
\end{aligned}
$$

Figure 4 illustrates this structure. We see that both vector fields have closed integral curves, and that the closed curves belonging to the two fields are 
homologous. This is a special case of the following result: If one family of integral curves of an almost product structure on the torus includes a band of the type illustrated in Figure 2, then any complementary family includes closed curves which are homologous to those of the first family. In fact, consider a region such as that bounded by $A A^{\prime} B^{\prime} B$ in Figure 4 . Such a region is topologically an annulus in the plane. Furthermore, if the curves are parametrized, we have integral curves entering this region across both boundaries (or leaving across both boundaries). Since the vector fields are nonsingular, the Poincaré-Bendixson theorem tells us there is a closed integral curve of the second field contained within the annulus. Again using nonsingularity this curve cannot be homologous to zero. Since it cannot cross itself anywhere it must be homologous to the boundary curves, that is, to the closed integral curves of the first field.

These examples have been analytic structures. There is no analytic structure having an infinite number of bands like those in Figure 1; however, a $C^{\infty}$ structure of this type may be constructed by the following method: Define a $C^{\infty}$ function $p(y)$ on the interval $0 \leqq y \leqq 1$ which is zero with all its derivatives at both endpoints, positive at interior points, and grows as $\exp (-1 / y)$ near the origin. Such a function is constructed in the book of Steenrod [27, pp. 25-26]. Let $q(y)=p(y) \sin (1 / y)$ in $[0,1]$, and extend to a $C^{\infty}$ function on the whole real line by periodicity. Then the desired almost product structure is given by:

$$
q(y) d y-d x=0, \quad q(y) d x+d y=0 .
$$

This example was worked out in cooperation with $\mathrm{C}$. W. Misner.

The above examples indicate that the pair of regular curve families defined by an almost product structure on the torus may involve almost any combination of the types of regular families; combination is restricted only in case one of the curve families includes a band like Figure 2 .

4. Potential theory on the torus. We shall now restrict ourselves to the case that the almost product structure of the torus can be represented by two pencils of parallel lines in the plane. In this situation, we can introduce a torsionless metric and compute specifically the operators $d^{\prime}, \delta^{\prime}$, and $\Delta^{\prime}$. This in turn will enable us to find explicitly the $d^{\prime}$ cohomology and the $\Delta^{\prime}$ harmonic space for the structure.

Let us suppose that the coordinates in the plane are $(r, s)$ and that a torus is given by $0 \leqq r \leqq 1,0 \leqq s \leqq 1$. Suppose an almost product structure is given such that local product coordinates $(x, y)$ at the point $(a, b)$ have the form $x=A(r-a)+C(s-b), \quad y=B(r-a)+D(s-b), \quad A D-B C \neq 0$.

Solving these equations, we may write

$$
\begin{aligned}
& r=\alpha x+\beta y+a \\
& s=\gamma x+\delta y+b
\end{aligned} \quad(\alpha \delta-\beta \gamma) \neq 0 .
$$


We define a metric by the equation $d s^{2}=d x^{2}+d y^{2}$ in terms of the local product coordinates. This definition is clearly valid throughout the torus, for the local coordinates at any other point may be gotten by translation. Then we have for a one form $\Phi=\phi(r, s) d x+\psi(r, s) d y$ the identities:

$$
\begin{aligned}
d^{\prime} \Phi & =\frac{d \psi}{\partial x} d x \wedge d y=\left(\alpha \frac{\partial \psi}{\partial r}+\gamma \frac{\partial \psi}{\partial s}\right) d x \wedge d y, \\
{ }^{*} \Phi & =-\psi d x+\phi d y \\
\delta^{\prime} \Phi & =-{ }^{*} d^{\prime *} \Phi=-\frac{\partial \phi}{\partial x} \\
\Delta^{\prime} \Phi & =-\frac{\partial^{2} \phi}{\partial x^{2}} d x-\frac{\partial^{2} \psi}{\partial x^{2}} d y .
\end{aligned}
$$

Suppose in particular that $\Phi=\phi(r, s) d x$. Then we have

$$
\Delta^{\prime} \Phi=-\frac{\partial^{2} \phi}{\partial x^{2}} d x=-\left(\alpha \frac{\partial}{\partial r}+\gamma \frac{\partial}{\partial s}\right)^{2} \phi d x .
$$

In order to compute the Green's operator for $\Delta^{\prime}$, we shall use an approach due to $\mathrm{K}$. Kodaira. He also derived the results here given for $\lambda$ irrational, where $\lambda=\gamma / \alpha$. First, we need the following lemma:

Lemma 4.1. $\phi$ is of class $C^{\infty}$ if and only if

$$
\sum_{m, n}\left(1+|m|^{2 p}\right)\left(1+|n|^{2 q}\right)\left|\phi_{m n}\right|^{2}<\infty, \quad \quad p, q=0,1, \cdots
$$

where $\phi$ is a square integrable function on the unit square and

$$
\phi=\sum_{m, n=-\infty}^{\infty} \phi_{m n} e^{2 \pi i(m r+n s)} .
$$

This is a long established result. If $\phi$ is $C^{\infty}$, the inequality may be proved by differentiation of the series. Conversely, if the inequality holds, we show that the series which is of the form of the derivative, converges to the derivative.

We are now prepared to examine $\Delta^{\prime}$ more closely, using the explicit formulas for $\Delta^{\prime} \Phi$ derived above. If $\lambda=\gamma / \alpha$ is irrational, then $m \alpha+n \gamma \neq 0$ for $(m, n)$ $\neq(0,0)$. Expanding $\phi$ in its Fourier series, we compute

$$
\Delta^{\prime} \Phi=4 \pi^{2} \sum_{(m, n) \neq(0,0)}(m \alpha+n \gamma)^{2} \phi_{m n} e^{2 \pi i(m r+n s)} d x,
$$

where $\Phi=\phi d x$. Since $m \alpha+n \gamma \neq 0, \Delta^{\prime} \phi=0$ if and only if $\phi=\phi_{00}$ is a constant. If $H^{\prime}$ is the projection operator on the harmonic space, then $H^{\prime} \Phi=\phi_{00} d x$, while 


$$
\Phi-H^{\prime} \Phi=\sum_{(m, n) \neq(0,0)} \phi_{m n} e^{2 \pi i(m r+n s)} .
$$

Next we wish to find $\Psi=\psi d x$ such that $\Delta^{\prime} \Psi=\Phi-H^{\prime} \Phi$; that is, $\Delta^{\prime} \psi=\phi-\phi_{00}$. Assume there exists such a $\psi$. Then, using the explicit formula for $\Delta^{\prime} \psi$ and equating coefficients in the Fourier series, we find for each $(m, n) \neq(0,0)$

$$
\psi_{m n}=\frac{\phi_{m n}}{4 \pi^{2}(m \alpha+n \gamma)^{2}}=\frac{\phi_{m n}}{4 \pi^{2} \alpha^{2}(m+n \lambda)^{2}},
$$

while $\psi_{00}$ is arbitrary; let us choose $\psi_{00}=0$. If we can show that $\sum \psi_{m n}$ converges, we will have constructed the Green's operator, while if it fails to converge, the Green's operator does not exist, in the sense that there is no $\psi$ in $\mathfrak{L}_{2}$ satisfying $\Delta^{\prime} \psi=\phi-H^{\prime} \phi$.

Let us suppose first that $|m+n \lambda| \geqq k / f(|n|)$, where $f(n)=O\left(n^{s}\right)$ for some integer $s>2$. By a theorem of Khintchine $\left(^{3}\right)$, this inequality holds for almost all $\lambda$. Then we have

$$
\left|\psi_{m n}\right|=\left|\frac{\phi_{m n}}{4 \pi^{2} \alpha^{2}(m+n \lambda)^{2}}\right| \leqq \frac{\left|\phi_{m n}\right| f(|n|)}{4 \pi^{2} \alpha^{2} k} \leqq k^{\prime}\left|\phi_{m n}\right||n|^{s} .
$$

Hence $\left|\psi_{m n}\right|^{2} \leqq k^{\prime \prime}|n|^{2 s}\left|\phi_{m n}\right|^{2} \leqq k^{\prime \prime}\left(1+|n|{ }^{2 s}\right)\left|\phi_{m n}\right|^{2}$, so that the series for $\psi$ converges, using Lemma 4.1 and the fact that $\phi$ is $C^{\infty}$. Using this last inequality, we get an inequality for

$$
\sum\left(1+|m|^{2 p}\right)\left(1+|n|^{2 q}\right)\left|\psi_{m n}\right|^{2} \text { by which we show that } \psi \text { is } C^{\infty} .
$$

Next consider the case that $\lambda=1+\sum_{h=1}^{\infty} 1 / N_{1} \cdots N_{h}$ where $N_{i}$ are positive integers such that $N_{i+1}>\exp \left(N_{1} \cdots N_{h}\right)$. In this case, we shall construct infinitely many linearly independent $\phi$ of $C^{\infty}$ for which $\Delta^{\prime} \psi=\phi-H^{\prime} \phi$ has no solution.

Lemma 4.2. Let $n_{h}=N_{1} \cdots N_{h}$. Then $n_{h} \lambda=m_{h}+\rho_{h}$ where $m_{h}$ is an integer and $0<\rho_{h}<2 \exp \left(-n_{h}\right)$.

In fact, by the definition of $\lambda, n_{h} \lambda=m_{h}+\sum_{k=h+1}^{\infty} 1 / N_{h+1} \cdots N_{k}$.

Now we define $\phi$ as follows:

$$
\phi_{m n}= \begin{cases}0 & \text { for }(m, n) \neq\left(-m_{h}, n_{h}\right), \\ c_{h} \exp \left(-n_{h} / 2\right) & \text { for }(m, n)=\left(-m_{h}, n_{h}\right)\end{cases}
$$

where $0<a<\left|c_{h}\right|<b<\infty$. To show that $\phi$ is $C^{\infty}$, consider

$$
\begin{aligned}
\sum_{m, n}\left(1+|m|^{2 p}\right)\left(1+|n|^{2 q}\right)\left|\phi_{m n}\right|^{2} & \leqq \sum_{n_{h}}\left(1+\left|m_{h}\right|^{2 p}\right)\left(1+\left|n_{h}\right|^{2 q}\right) b^{2} \exp \left(-n_{h}\right) \\
& \leqq k \sum_{n_{h}}\left(1+\left|n_{h}\right|^{2 p+2 q}\right) \exp \left(-n_{h}\right)<\infty .
\end{aligned}
$$

$\left(^{3}\right)$ See Koksma [15, pp. 47-49], for a proof of this theorem. 
Suppose $\psi$ exists. Then

$$
\psi_{-m_{h}, n_{h}}=\frac{1}{4 \pi^{2} \alpha^{2}\left(-m_{h}+n_{h} \lambda\right)^{2}} c_{h} \exp \left(-n_{h} / 2\right)
$$

so that

$$
\left|\psi_{-m_{h}, n_{h}}\right| \geqq \frac{a}{16 \pi^{2} \alpha^{2}} \exp \left(3 n_{h} / 2\right) \rightarrow \infty .
$$

Hence $\sum \psi_{m n} \exp (2 \pi i(m r+n s))$ fails to converge, so that the Green's operator is not defined for such a $\phi$. On the other hand, it does exist for all $\phi$ which may be written in the form $\phi=\sum_{m, n=-N}^{N} \phi_{m n} \exp (2 \pi i(m r+n s))$. Such $\phi$ are dense in $\mathfrak{L}_{2}$, for by truncating the Fourier series of a real function in the manner indicated, another real function is obtained. It is clear that the Green's operator $G^{\prime}$ is defined for such $\phi$ on account of the finiteness of the series. This shows that $G^{\prime}$ is an unbounded operator, since a bounded densely defined operator may be extended to all of $\mathfrak{L}_{2}$.

Next suppose that $\lambda$ is rational, so that $\lambda=-M / N$ in lowest terms. Let $\phi$ be $C^{\infty}$ and suppose $\Delta^{\prime} \phi=0$. Looking at the explicit expression for $\Delta^{\prime} \phi$, we see that $\phi_{m n}=0$ for $(m, n) \neq(k M, k N)$, while $\phi_{m n}$ is arbitrary for the remaining terms. Hence $\phi=\sum_{k=-\infty}^{\infty} \phi_{k} \exp (2 \pi i(M r+N s) k)$. Recalling that $\lambda=\gamma / \alpha$ $=-M / N$, let us write $\gamma=L M, \alpha=-L N$ and seek an interpretation for the $\Delta^{\prime}$ harmonic forms. We have

$$
\begin{aligned}
M r+N s & =L^{-1}(\gamma r-\alpha s)=L^{-1}[(\gamma \beta-\alpha \delta) y+(\gamma a-\alpha b)] \\
& =(M \beta+N \alpha) y+(M a+N b) .
\end{aligned}
$$

We have made use of the formula at the beginning of this section for transforming from $(r, s)$ to $(x, y)$ coordinates, noting that $\alpha \delta-\beta \gamma \neq 0$. This computation shows that $\phi$ is a function of $y$ alone, and is periodic of period $1 /(M \beta+N \alpha)$. Conversely, any function of $y$ with this period is expandable in a series of the above type, and so is $\Delta^{\prime}$ harmonic. (This suggests the interpretation that if $\lambda$ is irrational, the $\Delta^{\prime}$ harmonic forms are periodic of arbitrarily small periods, so constant.)

By an argument used before, if $\phi$ is any real function, the function $H^{\prime} \phi$ $=\sum_{k=-\infty}^{\infty} \phi_{k M, k N} \exp (2 \pi i(M r+N s) k)$ is also a real function. Restricting ourselves to the space of $\phi-H^{\prime} \phi$, and letting $\Delta^{\prime} \psi=\phi$, we find as before that

$$
\begin{aligned}
& \psi_{m n}=\frac{\phi_{m n}}{4 \pi^{2} \alpha^{2}(m+n \lambda)^{2}} \text { for }(m, n) \neq(k M, k N), \\
& \psi_{k M, k N} \text { is arbitrary, say zero. }
\end{aligned}
$$

Then $G^{\prime}$ will be defined if and only if 


$$
\sum_{(m, n) \neq k(M, N)} \frac{\phi_{m n}}{4 \pi^{2} \alpha^{2}(m+n \lambda)^{2}}
$$

converges. By number theory, we know that if $|n|>N>0$ and $n \neq k N$, then $|m+n \lambda| \geqq 1 / N$. Thus

$$
\left|\frac{\phi_{m n}}{4 \pi^{2} \alpha^{2}(m+n \lambda)^{2}}\right| \leqq \frac{N}{4 \pi^{2} \alpha^{2}}\left|\phi_{m n}\right| \quad \text { for }|n|>N,
$$

so that the series converges and $G^{\prime}$ is defined.

This analysis has been for form of type $(1,0)$. For an arbitrary form, the same analysis may be carried out, since $\Delta^{\prime}$ acts component-wise.

To complete this section, let us look briefly at the $d^{\prime}$ cohomology. Suppose $\Phi=\phi d x$, with $\phi=\sum \phi_{m n} \exp (2 \pi i(m r+n s))$; then $d^{\prime} \Phi=0$ automatically. If $\phi d x=d^{\prime} f=\partial f / \partial x d x$, where $f=\sum f_{m n} \exp (2 \pi i(m r+n s))$, then

$$
\phi_{m n}=2 \pi i(m \alpha+n \gamma) f_{m n} .
$$

If $\lambda$ is irrational, we have

$$
\begin{aligned}
& f_{m n}=\frac{\phi_{m n}}{2 \pi i \alpha(m+n \lambda)} \quad \text { for }(m, n) \neq(0,0), \\
& f_{00} \quad \text { is arbitrary. }
\end{aligned}
$$

If $\lambda$ is of the first type discussed, the series for $f$ converges, so that any $\Phi$ with $\phi_{00}=0$ satisfies $\Phi=d^{\prime} f$; consequently, the cohomology group is one dimensional. If $\lambda$ is of the second type, and $\phi$ is as in the discussion of that type, then $\sum f_{m n} \exp (2 \pi i(m r+n s))$ diverges; hence, the cohomology group is infinite dimensional. If $\lambda$ is rational, $f_{m n}$ is arbitrary for $(m, n)=(k M, k N)$, the series for $f$ converges, and the cohomology group is infinite dimensional. We may summarize these results in a table valid for forms of type $(1,0)$ :

\begin{tabular}{l|c|c|c}
$\lambda$ & $\begin{array}{c}\text { dimension of } \\
d^{\prime} \text { cohomology }\end{array}$ & $\begin{array}{c}\text { dimension of } \Delta^{\prime} \\
\text { harmonic space }\end{array}$ & $\begin{array}{c}\text { does } G^{\prime} \text { exist } \\
\text { everywhere in } \mathscr{L}_{2}\end{array}$ \\
\hline $\begin{array}{l}\text { rational } \\
\text { irrational I }\end{array}$ & $\infty$ & $\infty$ & yes \\
irrational II & 1 & 1 & yes \\
no
\end{tabular}

The example of the torus thus shows that even if the metric is torsionless, the Green's operator need not exist in the sense of $\mathfrak{L}_{2}$. Furthermore, the $\Delta^{\prime}$ harmonic space need not be isomorphic to the $d^{\prime}$ cohomology group; as we shall see later, the isomorphism does hold on any compact manifold on which the Green's operator is defined everywhere on $\mathscr{L}_{2}$.

5. The operator $\tilde{\Delta}$. The purpose of this paragraph is to compare $\Delta$ with $\tilde{\Delta}$, and to explore the properties of the latter operator. Both these operators are positive definite; in fact, they have precisely the same second order terms. 
$\tilde{\Delta}$ commutes with type projection operators, while $\Delta$ does not in general. On the other hand, $\Delta$ commutes with $d$ and $\delta$, while $\tilde{\Delta}$ fails to commute with $d, \delta, d^{\prime}, \delta^{\prime}$, or any of the other related differential operators. $\tilde{\Delta}$ has an inverse $\tilde{G}$, which also fails to commute with these operators. The solutions of $\tilde{\Delta} \phi=0$ are precisely those forms, each type component of which is harmonic in the ordinary sense. It is assumed that the manifold $V$ is compact and $C^{\infty}$.

We begin by finding conditions under which $\Delta$ equals $\tilde{\Delta}$.

Proposition 5.1. The following statements are equivalent:

(i) $\Delta=\tilde{\Delta}$.

(ii) $\Delta$ commutes with type projections.

(iii) $R_{i j}=0$ whenever $i$ and $j$ are of different types, while $R_{h i j k}=0$ whenever precisely three indices are of one type, or whenever $h$ and $i$ are of one type and $j$ and $k$ of the other.

Clearly, the first statement implies the second. To show that the second and third are equivalent, use the explicit expression

$$
\begin{aligned}
(\Delta \phi)_{i_{1}} \cdots i_{p}= & -g^{i j D_{i} D_{j} \phi_{i_{1}} \cdots i_{p}}-\sum_{\mu=1}^{p} R_{i_{\mu}}^{h} \phi_{i_{1} \cdots i_{\mu-1} h i_{\mu+1} \cdots i_{p}} \\
& -(1 / 2) \sum_{\mu, \nu=1}^{p} R_{i_{\mu} i_{\nu}}{ }^{h i} \phi_{i_{1} \cdots i_{\mu-1} h i_{\mu+1} \cdots i_{\nu-1} i i_{\nu+1} \cdots i_{p}}
\end{aligned}
$$

where $D_{i}$ is covariant differentiation with respect to the $i$ th variable. This expression may be found in the book of de Rham [20, p. 131], or that of Schiffer and Spencer [22, p. 418]. Assume $\Delta$ commutes with type and let $\phi$ be a $(1,0)$ form; examination of this explicit expression for $\Delta \phi$ shows that $R_{i j}=0$ unless $i$ and $j$ are of the same type. Next let $\phi$ be of type $(2,0)$ or $(0,2)$; using the result already obtained for $R_{i j}$, we can then show that the assertion about $R_{h i j k}$ is true. It then follows that the expressions for $\Delta \phi, \phi$ being of arbitrary type, preserve type; this proves the converse. It may be noted that whenever Proposition 5.1 holds, if $i_{1}$ and $i_{2}$ are of the first type and $j_{1}$ and $j_{2}$ of the second type, then $R_{i_{1} j_{1} i_{2} j_{2}}=R_{i_{1} j_{2} i_{2} j_{1}}$; this follows from the identity on cyclic permutation of the last three indices. The proof of proposition 5.1 will be complete as soon as we prove:

Lemma 5.1. $\tilde{\Delta}=\sum_{t, u} \prod_{t, u} \Delta \prod_{t, u}$.

In fact, $\Delta=d \delta+\delta d=\tilde{\Delta}+\left(d^{\prime} \delta^{\prime \prime}+\delta^{\prime \prime} d^{\prime}\right)+\left(d^{\prime \prime} \delta^{\prime}+\delta^{\prime} d^{\prime \prime}\right)$, the three operators being of types $(0,0),(1,-1)$, and $(-1,1)$ respectively. Thus if $\phi$ is of type $(t, u)$, we see that $\tilde{\Delta} \phi=\prod_{t, u} \Delta \phi$. But

$$
\left(\tilde{\Delta} \sum \prod_{t, u} \phi\right)=\sum \tilde{\Delta} \prod_{t, u} \phi=\sum \prod_{t, u} \Delta \prod_{t, u} \phi .
$$

Proposition 5.1 is a purely local theorem. It holds, in particular, if the 
metric is torsionless, for then statement (iii) is trivially true.

We turn now to the relation between $\Delta$ and $\tilde{\Delta}$ in the general case.

Lemma 5.2. For a form $\phi^{t, u}$ of pure type, $\Delta \phi=0$ if and only if $\Delta^{\prime} \phi=0$ and $\Delta^{\prime \prime} \phi=0$. If $\phi$ is not of pure type, $\Delta^{\prime} \phi=\Delta^{\prime \prime} \phi=0$ implies $\Delta \phi=0$, but not conversely. $\Delta^{\prime} \phi=\Delta^{\prime \prime} \phi=0$ implies that each $\prod_{t, u} \phi$ is harmonic.

In fact, $\Delta \phi=0$ implies that $d \phi=\delta \phi=0$, hence that $d^{\prime} \phi=d^{\prime \prime} \phi=\delta^{\prime} \phi=\delta^{\prime \prime} \phi=0$ on account of pureness of type; this means $\Delta^{\prime} \phi=\Delta^{\prime \prime} \phi=0$. The converse is proved by simply reversing the steps. If $\phi$ is not pure in type, the converse is still true, but the direct argument fails. The last statement holds by virtue of the type commutation of $\Delta^{\prime}$ and $\Delta^{\prime \prime}$.

Proposition 5.2. Let $\phi$ be a pure form of type $(t, u)$. Then the following are equivalent statements:

(i) $\tilde{\Delta} \phi=0$.

(ii) $\prod_{t, u} \Delta \phi=0$.

(iii) $d \phi=\delta \phi=0$.

(iv) $d^{\prime} \phi=\delta^{\prime} \phi=d^{\prime \prime} \phi=\delta^{\prime \prime} \phi=0$.

(v) $\Delta^{\prime} \phi=\Delta^{\prime \prime} \phi=0$.

(vi) $\Delta \phi=0$.

In fact, (i) implies (ii) by Lemma 5.1. (ii) implies (iii) because 0 $=\left(\prod_{t, u} \Delta \phi, \phi\right)=(\Delta \phi, \phi)=(d \phi, d \phi)+(\delta \phi, \delta \phi)$; hence $d \phi=\delta \phi=0$. (iii) implies (iv) because $d^{\prime} \phi$ and $d^{\prime \prime} \phi$ are of different types. That (iv) implies (v) is obvious, that (v) implies (vi) is Lemma 5.2, and that (vi) implies (i) follows from Lemma 5.1.

Let us introduce the following notations:

$H^{p}=\{\phi \mid \phi$ is of degree $p, \Delta \phi=0\}$,

$H^{t, u}=\{\phi \mid \phi$ is of type $(t, u), \Delta \phi=0\}$,

$\bar{H}^{p}=\left\{\phi \mid \phi\right.$ is of degree $p, \phi=\sum \phi^{t, u}, \Delta \phi=0$, but for each $(t, u)$ either $\Delta^{\prime} \phi^{t, u} \neq 0$ or $\left.\Delta^{\prime \prime} \phi^{t, u} \neq 0\right\}=\left\{\phi \mid \phi\right.$ is of degree $p, \Delta \phi=0, \Delta \phi^{t, u} \neq 0$ for all $\left.(t, u)\right\}$. It is then clear that $H^{p}=\bar{H}^{p} \oplus \sum_{t, u} H^{t, u}$, where each sum is direct. Since $H^{p}$ is finite dimensional, so are $\bar{H}^{p}$ and $H^{t, u}$.

$$
\check{H}^{p}=\{\phi \mid \phi \text { is of degree } p, \tilde{\Delta} \phi=0\} .
$$

We wish to show that $H^{p}$ is the direct sum of $\widetilde{H}^{p}$ and $\bar{H}^{p}$. First, notice that $\widetilde{H}=\left\{\phi \mid \Delta^{\prime} \phi=\Delta^{\prime \prime} \phi=0\right\}$, or otherwise stated, $\tilde{\Delta} \phi=0$ if and only if $\Delta^{\prime} \phi$ $=\Delta^{\prime \prime} \phi=0$. For pure forms, this is obvious by Proposition 5.2. Since however, $\tilde{\Delta}, \Delta^{\prime}$, and $\Delta^{\prime \prime}$ all commute with type, the statement immediately extends to all forms. Next, clearly $\tilde{H}^{p}$ is contained in $H^{p}$; for $\tilde{\Delta} \sum \phi^{t, u}=0$ implies $\tilde{\Delta} \phi^{t, u}=0$, which implies $\Delta \phi^{t, u}=0$, so $\Delta \phi=0$. The inverse containment is false in general. Again making use of Proposition 5.2, it is now proved that:

Theorem 5.1. $\tilde{H}=\sum_{t, u} H^{t, u}$, so that $H^{p}=\tilde{H}^{p} \oplus \bar{H}^{p}$. 
Having established the relationship between $\Delta$ and $\tilde{\Delta}$, we now turn to the study of the latter operator. Our immediate purpose is to establish the existence of the Green's operator $\tilde{G}$ for $\tilde{\Delta}$. We must begin by computing explicitly the appearance of the operator $\tilde{\Delta}$ in a local product coordinate system. To this end, as well as for later use, we compute $\Delta^{\prime}$. The computation follows closely that for the operator $\Delta$, for which see de Rham [19, pp. 127$131]$.

Let

$$
\phi=\sum_{(i)(j)} \phi_{i_{1}} \cdots i_{r} j_{1} \cdots j_{s} d x^{i_{1}} \wedge \cdots \wedge d x^{i_{r}} \wedge d y^{j_{1}} \wedge \cdots \wedge d y^{j_{s}} .
$$

Then

$$
\left(d^{\prime} \phi\right)_{k_{1} \cdots k_{r+1} j_{1} \cdots j_{s}}=\sum_{i(i)} \delta_{k_{1} \cdots k_{r+1}}^{i i_{1} \cdots i_{r}} \frac{\partial \phi_{i_{1} \cdots i_{r} j_{1} \cdots j_{s}}}{\partial x^{i}} .
$$

Suppose $\psi$ is a form with compact carrier contained in the coordinate neighborhood. By writing out explicitly the equation $\left(\delta^{\prime} \phi, \psi\right)=\left(\phi, d^{\prime} \psi\right)$ and making use of the formula for $d^{\prime} \psi$, we find that

$$
\left(\delta^{\prime} \phi\right)^{i \cdots i_{r} j_{1} \cdots j_{s}}=\sum_{i_{1}}-g^{-1 / 2} \partial / \partial x^{i_{1}}\left(g^{1 / 2} \phi^{i_{1} \cdots i_{r} j_{1} \cdots j_{s}}\right)
$$

where $g$ is the determinant of the matrix $\left(g_{i j}\right)$. To get the principal part of $\Delta^{\prime} \phi$, we may assume that the $g_{i j}$ are constants. Then

$$
\left(\delta^{\prime} \phi\right)_{k_{2}} \cdots k_{r} l_{1} \cdots l_{s}=-g^{\prime i k} \partial / \partial x^{i} \phi_{k k_{2}} \cdots k_{r} l_{1} \cdots l_{s} \text {. }
$$

Recalling that $d^{\prime} \delta^{\prime}+\delta^{\prime} d^{\prime}=\Delta^{\prime}$, using these expressions for $d^{\prime}$ and $\delta^{\prime}$ and the identity

$$
-\delta_{k m_{1} \cdots m_{r}}^{j k_{1} \cdots k_{r}}+\delta_{k}^{j} \delta_{m_{1} \cdots m_{r}}^{k_{1} \cdots k_{r}}=\sum_{(n)} \delta_{m_{1} \cdots m_{r}}^{j n_{1} \cdots n_{r-1}} \delta_{k n_{1} \cdots n_{r-1}}^{k_{1} \cdots k_{r}}
$$

we find that

$$
\left(\Delta^{\prime} \phi\right)_{m_{1} \cdots m_{r} l_{1} \cdots l_{s}}=-g^{\prime i j} \frac{\partial^{2}}{\partial x^{i} \partial x^{j}} \phi_{m_{1} \cdots m_{r} l_{1} \cdots l_{s}}+\cdots,
$$

where the missing terms involve first and zero order derivatives of all components of $\phi$.

In case the metric is torsionless, we may replace $\partial / \partial x^{i}$ by covariant differentiation, and thus compute $\Delta^{\prime}$ explicitly. We find that

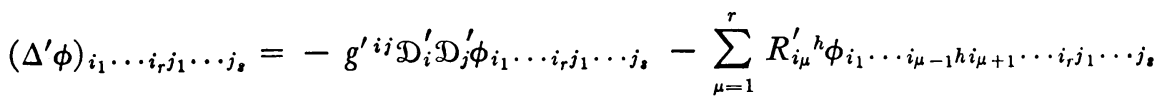

$$
\begin{aligned}
& -(1 / 2) \sum_{\mu, \nu=1}^{r} R_{i \mu i_{\nu}}{ }^{i} \phi_{i_{1}} \cdots i_{\mu-1} h i_{\mu+1} \cdots i^{\nu}-i^{i} i^{\nu}+1 \cdots i_{r} j_{1} \cdots j_{\varphi} \cdot
\end{aligned}
$$


Here $D_{i}^{\prime}$ is covariant differentiation with respect to $x^{i}$, while $R_{\alpha \beta}^{\prime}$ and $R_{\alpha \beta \gamma \delta}^{\prime}$, are zero unless all indices are of the first type.

Since $\tilde{\Delta}=\Delta^{\prime}+\Delta^{\prime \prime}$, it is now clear that

$$
\tilde{\Delta}=-g^{\prime i j} \frac{\partial^{2}}{\partial x^{i} \partial x^{j}}-g^{\prime \prime i j} \frac{\partial^{2}}{\partial y^{i} \partial y^{i}}+\text { lower order terms. }
$$

To carry out the construction of $\tilde{G}$, we apply the method of Spencer [26, pp. 25-31]. We shall say that $\phi$ is in the domain of $\tilde{\Delta}$ if $\phi$ is in the domain of (the closure of) $d^{\prime}, \delta^{\prime}, d^{\prime \prime}$, and $\delta^{\prime \prime}, d^{\prime} \phi$ is in the domain of (the closure of) $\delta^{\prime}$, etc. $\left.{ }^{4}\right)$ Let $\widetilde{D}_{s}(\phi, \psi)=\left(d^{\prime} \phi, d^{\prime} \psi\right)+\left(\delta^{\prime} \phi, \delta^{\prime} \psi\right)+\left(d^{\prime \prime} \phi, d^{\prime \prime} \psi\right)+\left(\delta^{\prime \prime} \phi, \delta^{\prime \prime} \psi\right)+s(\phi, \psi)$, for $s \geqq 0$. If $\phi$ and $\psi$ are in the domain of $\tilde{\Delta}$, then $\widetilde{D}(\phi, \psi)=\widetilde{D}_{0}(\phi, \psi)=(\phi, \tilde{\Delta} \psi)$ $=(\tilde{\Delta} \phi, \psi)$. Since $V$ is compact, the analog of Spencer's space $G$ is simply the domain of $\tilde{\Delta}$. Given $\beta$ in $\mathfrak{L}_{2}(V)$, define $\widetilde{E}_{s}(\phi, \psi)=\tilde{D}(\phi, \psi)+s(\phi-\gamma, \psi-\gamma)$, where $\gamma=\beta / s$. By minimizing $\tilde{E}_{s}(\phi)=\tilde{E}_{s}(\phi, \phi)$ over all $\phi$ in its domain, we obtain a form $\alpha$ satisfying $\widetilde{D}_{s}(\alpha, \phi)=(\beta, \phi)=\left(\alpha, \tilde{\Delta}_{s} \phi\right)$, where $\tilde{\Delta}_{s}=\tilde{\Delta}+s$. Since $\tilde{\Delta}=-g^{i j} \partial^{2} / \partial z^{i} \partial z^{i}+$ lower order terms, with $g^{i j}$ positive definite, we may apply the method of Kodaira [14] to construct a parametrix and prove a differentiability lemma. (To define and use the parametrix instead of the elementary solution, certain modifications of the kind discussed in $\S 6$ are required.) We may then conclude that $(\tilde{\Delta}+s) \alpha=\tilde{\Delta}_{s} \alpha=\beta$, if $\beta$ is $C^{\infty}$. Define $\widetilde{G}_{s}$ by the equation $\widetilde{G}_{s} \beta=\alpha$; then it is a symmetric bounded linear transformation on $\mathfrak{L}_{2}(V)$, satisfying $\widetilde{D}_{s}\left(\widetilde{G}_{s} \beta\right) \leqq 1 / s\left|\widetilde{G}_{s} \beta\right|^{2}$, where || denotes the norm in $\mathscr{L}_{2}(V)$. $\widetilde{G}_{s} \beta$ is in the domain of $\tilde{\Delta}$, and $\tilde{G}_{s} \tilde{\Delta}_{s} \phi=\widetilde{\Delta}_{s} \widetilde{G}_{s} \phi=\phi . \tilde{\Delta}$ is self-adjoint, since $\widetilde{G}_{s}$ $=\left(\tilde{\Delta}_{s}\right)^{-1}$ is. $\widetilde{G}_{s}$ is completely continuous, and $\widetilde{H}=\left\{\phi \mid \phi-s \widetilde{G}_{s} \phi=0\right\}$. Hence, there is a unique solution of $\psi-s \widetilde{G}_{s} \psi=\beta$ orthogonal to $\tilde{H}$. Define $\tilde{G} \beta=\widetilde{G}_{s} \psi$. $\widetilde{G}$ is the desired Green's operator. It satisfies $\beta=\tilde{\Delta} \widetilde{G} \beta+\widetilde{H} \beta=\tilde{G} \tilde{\Delta} \beta+\tilde{H} \beta$ where $\tilde{H} \beta$ is the projection of $\beta$ on the space $\widetilde{H}$. Furthermore, $\widetilde{G} \widetilde{H}=\widetilde{H} \widetilde{G}=0$. $\widetilde{G}$ is symmetric and completely continuous.

$\widetilde{G}$ fails to commute with $d, d^{\prime}$, and the other related operators; this prevents our proving an analog to Hodge's theorem. In fact, we have the following:

Proposition 5.3. Let $A$ be an operator such that $\tilde{H} A=A \widetilde{H}=0$. Then $\tilde{G} A-A \tilde{G}=\tilde{G}(A \tilde{\Delta}-\tilde{\Delta} A) \tilde{G}$.

Since $\tilde{H}(\tilde{G} A-A \tilde{G})=0$, we have $\tilde{G} A-A \tilde{G}=\tilde{G} \tilde{\Delta}(\tilde{G} A-A \tilde{G})=\tilde{G} A-\tilde{G} \tilde{\Delta} A \tilde{G}$ $=\tilde{G} A-\tilde{G} A \tilde{\Delta} \tilde{G}+\tilde{G} A \tilde{\Delta} \widetilde{G}-\tilde{G} \tilde{\Delta} A \tilde{G}$. Since $\tilde{G} A \tilde{\Delta} \tilde{G}=\tilde{G} A(I-\tilde{H})=\tilde{G} A$ where $I$ is the identity operator, we get $\widetilde{G} A-A \widetilde{G}=\widetilde{G} A \tilde{\Delta} \widetilde{G}-\tilde{G} \tilde{\Delta} A \tilde{G}$.

By reason of this proposition, we need only show that $d^{\prime}, \delta^{\prime}, d^{\prime \prime}, \delta^{\prime \prime}, \Delta^{\prime}$, $\Delta^{\prime \prime}, d, \delta$, and $\Delta$ are orthogonal to $\tilde{H}$ and fail to commute with $\tilde{\Delta}$. These demonstrations are trivial.

This paragraph has established that $\Delta=\tilde{\Delta}$ if and only if $\Delta$ commutes with

(4) For the definition and properties of the closure of an unbounded operator in Hilbert space, see Riesz and Nagy [21, Chapter viii]. 
type, that $\tilde{H}$ is the space which consists of all ordinary harmonic forms of pure type, and that $\tilde{\Delta}$ has an inverse $\tilde{G}$ which fails to commute with the differential operators we are concerned with. In the concluding paragraph, we shall discuss $\Delta^{\prime}$, which differs from the usual Laplacians by being semidefinite. For $\Delta^{\prime}$, the operators $G_{s}^{\prime}$ may be defined, but are not completely continuous; thus the construction used here to get $\widetilde{G}$ fails, and in fact as we have seen, $G^{\prime}$ does not always exist in the sense of being everywhere defined in $\mathfrak{L}_{2}$. It does exist as a densely defined operator; the next paragraph will prove this as well as other properties of $G^{\prime}$ and $\Delta^{\prime}$.

6. Green's operator for $\Delta^{\prime}$. Let $D_{x}^{r}$ be the sheaf of germs of $r$ forms which depend on $x$ alone, and similarly for $D_{y}^{s}$. A section $\phi$ of $D_{x}^{r}$ is expressible in a local product coordinate system as $\phi=\sum_{(i)} \phi_{i_{1}} \cdots i_{r}(x) d x^{i_{1}} \wedge \cdots \wedge d x^{i_{r}}$. Let $E$ be the direct sum $\sum_{r, s} D_{x}^{r} \wedge D_{v}^{s}$; then a section of $E$ has the local expression

$$
\phi=\sum_{r, s} \sum_{(i)(j)} \phi_{i_{1} \cdots i_{r}}(x) d x^{i_{1}} \wedge \cdots \wedge d x^{i_{r}} \wedge \psi_{j_{1}} \cdots j_{s}(y) d y^{i_{1}} \wedge \cdots \wedge d y^{j_{s}} .
$$

We shall show that in the case of a compact almost product manifold with torsionless metric, the Green's operator is defined for all $C^{\infty}$ sections of $E$. For this purpose, we shall define the notion of coherent subsheaf $B$ of $E$; by considering each $C^{\infty}$ section of $E$ as a section of some $B$, we construct the Green's operator, applying the method of Morrey and Eells [16, Chapter II], in order to do so. This approach to the problem was suggested by Professor D. C. Spencer.

A subsheaf $\left(S^{\prime}, \pi^{\prime}, X\right)$ of a sheaf $(S, \pi, X)$ is an open set $S^{\prime}$ in $S$, with projection $\pi^{\prime}$ which is $\pi$ restricted to $S^{\prime}$, such that the stalk $S_{x}^{\prime}$ is a subvectorspace of the stalk $S_{x}$. A coherent subsheaf $B$ of $E$ is a subsheaf such that given any point $p$ in $V$, there is a neighborhood $U_{p}$ and a set of forms $\gamma_{1}, \cdots, \gamma_{N}$ with $\gamma_{i}$ a section of $D_{\nu}^{s}$ over $U_{p}$, such that any $\phi$ in the stalk $B_{q}$ for $q$ in $U_{p}$ may be expressed as $\sum \phi_{i} \wedge \gamma_{i}$, this expression being valid in some $U_{q}$ contained in $U_{p}$. Here $N=N(p)$ and $s=s\left(\gamma_{i}\right)$. We might express this by saying that in any sufficiently small neighborhood, any section of $B$ depends upon a finite number of fixed forms in $y$; or more briefly, $B$ is finite in $y$.

Proposition 6.1. Let $\phi_{1}, \cdots, \phi_{t}$ be a finite set of sections of $E$. Then there exists a coherent subsheaf $B$ of $E$ such that $\phi_{1}, \cdots, \phi_{t}$ are sections of $B$.

Each $\phi_{i}$ is expressible as $\phi_{i}=\sum_{j} \phi_{i j} \wedge \gamma_{i j}$ in some sufficiently small neighborhood of any point $p$ of the manifold $V$. Take a neighborhood $U_{p}$ of $p$ small enough so that every $\phi_{i}$ is so expressible. $V$ being compact, it is covered by a finite number of such neighborhoods, say $\left\{U_{\alpha}\right\}$. We define $B$ as a sheaf of germs in the following way: Consider a basis $\left\{W_{\beta}\right\}$ of open sets of $V$ such that each $W_{\beta}$ is contained in some $U_{\alpha}$. If $W_{\beta}$ is contained in $U_{\mu} \cap \cdots \cap U_{\nu}$ but in no other sets of $\left\{U_{\alpha}\right\}$, take as the set of sections $F_{\beta}$ over $W_{\beta}$ the set 


$$
\left\{\phi \mid \phi=\sum_{r, k,(i)} \phi_{i_{1} \cdots i_{r}}(x) d x^{i_{1}} \wedge \cdots \wedge d x^{i_{r}} \wedge \gamma_{k}\right\}
$$

where $\gamma_{k}$ is one of the $\gamma_{i j}$ associated to one of the neighborhoods $U_{\mu}, \cdots, U_{\nu}$. If $W_{\alpha}$ is contained in $W_{\beta}$, we map $F_{\beta}$ into $F_{\alpha}$ by restriction; going to the direct limit, we get the desired sheaf $B$. The stalk of $B$ at $p$ consists of germs of the form $\sum_{k} \phi_{k} \wedge \gamma_{k}$, where $\gamma_{k}$ is any one of the $\gamma_{i j}$ defined in any $U_{\alpha}$ containing $p$.

We have thus established that any section of $E$ is also a section of some coherent $B$. Next we define certain function spaces associated to $B$ which will be useful in constructing the Green's operator. We shall be concerned with two inner products. The first, denoted by $(\omega, \phi)$, is defined as the usual inner product of forms on a manifold. The second is defined by

$$
((\omega, \phi)) \mathfrak{u}^{\prime}=(\omega, \phi)+\sum_{\alpha} \int_{U_{\alpha}} \sum_{(i)(j) i} \frac{\partial \omega_{i_{1}} \cdots i_{r_{j}} j_{1} \cdots j_{\varepsilon}}{\partial x^{i}} \frac{\partial \phi_{i_{1} \cdots i_{r} j_{1} \cdots j_{s}}}{\partial x^{i}} d x d y
$$

where $\mathfrak{U}$ is a finite covering $\left\{U_{\alpha}\right\}$. The subscript $\mathfrak{U}$ will usually be omitted. The space $\mathfrak{L}_{2}(B)$ is the completion in the first norm of the set of $C^{\infty}$ sections of $B$; the space $\mathcal{P}_{2}^{\prime}(B)$ is the completion in the second norm.

Proposition 6.2. Every form in $\mathfrak{L}_{2}(B)$ is expressible locally as $\sum \phi_{i} \wedge \gamma_{i}$, with the $\phi_{i}$ ordinary square integrable forms in $x$ and $\gamma_{i}$ the forms in $y$ generating the coherent sheaf. Forms in $\mathcal{P}_{2}^{\prime}(B)$ are expressible as $\sum \phi_{i} \wedge \gamma_{i}$ with $\gamma_{i}$ as before and $\phi_{i}$ forms in $\boldsymbol{P}_{2}$ in $x$ space.

The concept of $\boldsymbol{P}_{2}$ is defined and its properties given in Morrey and Eells [16]. This proposition states that the forms in $\mathscr{L}_{2}(B)$ may be expressed by means of the same forms $\gamma_{i}$ in $y$ as are used to express the $C^{\infty}$ sections of $B$; or that $\mathfrak{L}_{2}(B)$ is finite in $y$; and similarly for $\mathcal{P}_{2}^{\prime}(B)$. To prove it, let $\left\{\phi_{\mu}\right\}$ be a sequence of $C^{\infty}$ sections of $B$ such that $\left|\phi_{\mu}-\phi_{\nu}\right|^{2} \rightarrow 0$ as $\mu, \nu \rightarrow \infty$, where $|\alpha|^{2}$ $=(\alpha, \alpha)$. Then

$$
\left|\phi_{\mu}-\phi_{\nu}\right|^{2} \geqq \int_{U} \sum \Gamma^{(i)(k)(j)(l)}\left(\phi_{\mu}-\phi_{\nu}\right)_{(i)(j)}\left(\phi_{\mu}-\phi_{\nu}\right)_{(k)(l)} g^{1 / 2} d x d y
$$

where $U=U_{x} \times U_{y}$ is a local product coordinate neighborhood which is one of the defining neighborhoods for $B$, and

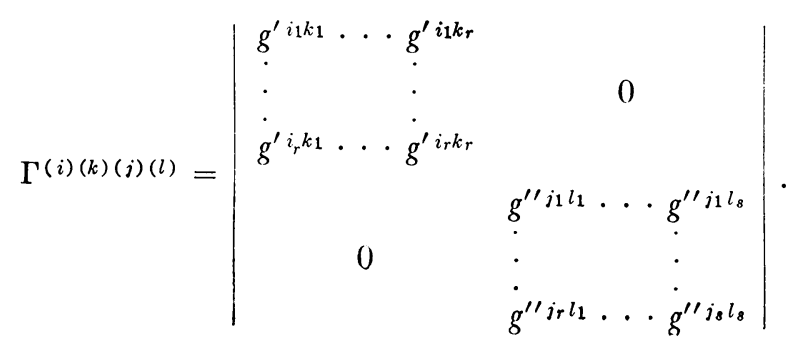


Hence $\left|\phi_{\mu}-\phi_{\nu}\right|^{2} \geqq K \int_{U} \sum_{(i)(j)}\left[\left(\phi_{\mu}-\phi_{\nu}\right)_{(i)(j)}\right]^{2} d x d y$, using the relation between the given Riemann metric and the Euclidean metric on the space $U$. We have on $U$ the relation $\psi=\sum \psi_{i} \wedge \gamma_{i}$ for all $\psi \in \Gamma(B, U)$. It may be assumed that

$$
\int_{U_{y}} \sum_{(j)} \gamma_{(j) j} \gamma_{(j) k} d y=\delta_{j k}
$$

for the $\gamma_{j}$ may be orthonormalized by the Gram-Schmidt process. Writing $\phi_{\mu}=\sum \phi_{u i} \wedge \gamma_{i}$, a formula valid for the restriction of $\phi_{\mu}$ to $U$, we have

$$
\begin{aligned}
& \left|\phi_{\mu}-\phi_{\nu}\right|^{2} \geqq K \int_{U} \sum_{(i)(j) j}\left[\left(\phi_{\mu}-\phi_{\nu}\right)_{(i) j} \gamma_{(j) j}\right]^{2} d x d y \\
& =K \sum_{(i)(j) j} \int_{U_{x}}\left[\left(\phi_{\mu}-\phi_{\nu}\right)_{(i) j}\right]^{2} d x \int_{U_{\nu}} \gamma_{(j) j}^{2} d y \\
& =K \sum_{(i) j} \int_{U_{x}}\left[\left(\phi_{\mu}-\phi_{\nu}\right)_{(i) j}\right]^{2} d x \\
& \rightarrow 0 \text {. }
\end{aligned}
$$

Thus each $\phi_{i}=\lim \phi_{\mu i}$ is in $\mathscr{L}_{2}\left(U_{x}\right)$ as asserted, and $\phi=\sum \phi_{i} \wedge \gamma_{i}$, valid in $U$. In particular, all functions in $\mathfrak{L}_{2}(B)$ are $C^{\infty}$ in $y$. Similar statements clearly hold for the Hilbert space $\mathcal{P}_{2}^{\prime}(B)$.

The following proposition is fundamental to our theory, just as it is in the work of Morrey and Eells [16]; its usefulness is that it enables us to minimize the Dirichlet integral and know immediately that the minimizing form is nonzero. The finiteness of $\mathscr{L}_{2}(B)$ in $y$ is an essential hypothesis.

Proposition 6.3. Let $\omega_{\beta} \rightarrow \omega$ weakly in $\rho_{2}^{\prime}(B)$. Then $\omega_{\beta} \rightarrow \omega$ strongly in $\mathfrak{L}_{2}(B)$.

Since clearly $\mathcal{P}_{2}^{\prime}(B)$ is a vector subspace of $\aleph_{2}(B)$, we know $\omega_{\beta}$ and $\omega_{\beta}$ are in $\mathfrak{L}_{2}(B)$. Let $\left\{U_{\alpha}\right\}$ be a finite covering of the manifold by coordinate cubes which are characteristic neighborhoods for the sheaf $B$. Since the integrand is everywhere positive, we have

$$
\begin{aligned}
& \int_{U_{\alpha}} \Gamma^{(i)(k)(j)(l)}\left(\omega_{\beta}-\omega\right)_{(i)(j)}\left(\omega_{\beta}-\omega\right)_{(k)(l)} g^{1 / 2} d x d y \\
& \leqq\left|\omega_{\beta}-\omega\right|^{2} \\
& \leqq \sum_{\alpha} \int_{U_{\alpha}} \Gamma^{(i)(k)(j)(l)}\left(\omega_{\beta}-\omega\right)_{(i)(j)}\left(\omega_{\beta}-\omega\right)_{(k)(l)} g^{1 / 2} d x d y .
\end{aligned}
$$

Hence, it is necessary and sufficient to prove convergence in each coordinate system. Using the relation between the norms in the metrics $g_{i j}$ and $\delta_{i j}$, we have 


$$
\begin{aligned}
K_{1} \int_{U_{\alpha}} \sum_{(i)(j)}\left[\left(\omega_{\beta}-\omega\right)_{(i)(j)}\right]^{2} d x d y \\
\quad \leqq \int_{U_{\alpha}} \Gamma^{(i)(k)(j)(l)}\left(\omega_{\beta}-\omega\right)_{(i)(j)}\left(\omega_{\beta}-\omega\right)_{(k)(l)} g^{1 / 2} d x d y \\
\quad \leqq K_{2} \int_{U_{\alpha}} \sum_{(i)(j)}\left[\left(\omega_{\beta}-\omega\right)_{(i)(j)}\right]^{2} d x d y \\
\quad=K_{2} \int_{U_{\alpha x}} \sum_{(i) i}\left[\left(\omega_{\beta}-\omega\right)_{(i) i}\right]^{2} d x
\end{aligned}
$$

where $\omega_{\beta}=\sum \omega_{\beta i} \wedge \gamma_{i}$ and $\omega=\sum \omega_{i} \wedge \gamma_{i}$, the $\gamma_{i}$ being orthonormal in $U_{\alpha}$. The final integrand is a finite sum because there are a finite number of $\gamma_{i}$. We have made use of Proposition 6.2. A lemma of Morrey and Eells [16, pp. 99$100]$, states that

$$
\int_{U_{\alpha^{x}}}\left|f(x)-f^{*}\right|^{2} d x \leqq p / 3 \sum_{k=1}^{p} h^{2} \int_{U_{\alpha x}}\left(\frac{\partial f}{\partial x^{k}}\right)^{2} d x
$$

where $f^{*}$ is the average value of $f$ over the $p$ cube of side $h$, that is over $U_{\alpha x}$, and $f$ is assumed to be in $\mathcal{P}_{2}^{\prime}$. More generally, if $U_{\alpha x}$ is cut up into smaller cubes of side $\epsilon$, and $\omega^{*}$ is the step function defined by taking the average of $\omega$ over each small cube, then

$$
\int_{U_{\alpha x}}\left|\omega_{(i) i}(x)-\omega_{(i) i}^{*}\right|^{2} d x \leqq p / 3 \epsilon^{2} \int_{U_{\alpha x}} \sum_{k=1}^{p}\left(\frac{\partial \omega_{(i) i}}{\partial x^{k}}\right)^{2} d x .
$$

Summing over ( $i$ ) and $i$, we get

$$
\int_{U_{\alpha x}} \sum_{(i) i}\left|\omega_{(i) i}(x)-\omega_{(i) i}^{*}\right|^{2} d x \leqq p / 3 \epsilon^{2}\|\omega\|^{\prime}
$$

where $\|\omega\|^{\prime}=((\omega, \omega))^{\prime}$; a similar equation holds for each $\omega_{\beta}$. $\|\omega\|^{\prime}$ and $\left\|\omega_{\beta}\right\|^{\prime}$ are uniformly bounded because of weak convergence in $\mathcal{P}_{2}^{\prime}(B)$; thus the right hand side may be made less than $\epsilon / 3$ independently of $\beta$. On the other hand, we have for the average values

$$
\int_{U_{\alpha x}} \sum_{(i) i}\left|\stackrel{*}{\omega_{\beta(i) i}}-\stackrel{*}{\omega_{(i) i}}\right|^{2} d x<\frac{\epsilon}{3}
$$

for $\beta$ sufficiently large, since the weak convergence of $\omega_{\beta i}$ to $\omega_{3}$ in $\mathcal{Q}_{2}^{\prime}(B) \mathrm{im}$ plies the weak convergence of $\omega_{\beta i}$ to $\omega_{\beta}$ in $\aleph_{2}\left(U_{\alpha x}\right)$, and this in turn implies strong convergence in $\mathscr{L}_{2}\left(U_{\alpha x}\right)$ of the average values. Combining these facts,

$$
\int_{U_{\alpha x}} \sum_{(i) i}\left|\omega_{(i) i}(x)-\omega_{\beta(i) i}(x)\right|^{2} d x<\epsilon
$$


for $\beta$ sufficiently large. This completes the proof of the proposition.

Next we define the Dirichlet integral $D^{\prime}(\omega)=\left(d^{\prime} \omega, d^{\prime} \omega\right)+\left(\delta^{\prime} \omega, \delta^{\prime} \omega\right) . d^{\prime}$ and $\delta^{\prime}$ are bounded operators from $\mathcal{P}_{2}^{\prime}(B)$ into $\mathscr{L}_{2}(B)$, while $D^{\prime}(\omega)$ is lower semicontinuous with respect to weak convergence in $P_{2}^{\prime}(B)$. This fact, combined with the lemmas about to be proved and with Proposition 6.3, will enable us to construct the Green's operator as the solution of a minimizing problem.

LEMMA 6.1. Let

$$
D_{0}^{\prime}(\omega)=\sum_{(i)(j) k} \int_{U}\left(\partial \omega_{(i)(j)} / \partial x^{k}\right)^{2} d x d y,
$$

while $\delta_{0}^{\prime}, \Delta_{0}^{\prime}$, and $(\phi, \psi)_{0}$ refer to $\delta^{\prime}, \Delta^{\prime}$, and $(\phi, \psi)$ computed in the euclidean norm. Let $\epsilon>0$ and let $x_{0}$ be a point in $V$. Then there is a coordinate sphere $U$ about $x_{0}$ and constants $k$ and $l$ such that

$$
k D^{\prime}(\omega) \geqq(1-\epsilon) D_{0}^{\prime}(\omega)-l(\omega, \omega)
$$

for any $\omega$ whose support is in $U$, where $\omega$ is of class $C^{\infty}$.

Lemma 6.2. For each coordinate covering $\mathfrak{u}$ there exist constants $K \mathfrak{u}>0$ and $L_{\mathfrak{u}}$ such that

$$
D^{\prime}(\omega) \geqq K \mathfrak{u}\|\omega\| \mathfrak{u}-L \mathfrak{u}(\omega, \omega)
$$

Lemma 6.1 is proved by a method of Gaffney [9]. By Stokes formula, using the fact that $\omega$ vanishes on the boundary, we show easily that

$$
\left(\Delta_{0}^{\prime} \omega, \omega\right)_{0}=\left(d^{\prime} \omega, d^{\prime} \omega\right)_{0}+\left(\delta_{0}^{\prime} \omega, \delta_{0}^{\prime} \omega\right)_{0}=D_{0}^{\prime}(\omega) .
$$

Using a linear transformation consistent with the local product structure, we may change coordinates in the neighborhood of $x_{0}$ so that $g_{i j}(0)=\delta_{i j}$. Then

$$
\begin{aligned}
D_{0}^{\prime}(\omega) & =\left|d^{\prime} \omega\right|_{0}^{2}+\left|\delta_{0}^{\prime} \omega\right|_{0}^{2} \leqq C_{1}\left[\left|d^{\prime} \omega\right|^{2}+\left|\delta_{0}^{\prime} \omega\right|^{2}\right] \\
& \leqq 2 C_{1}\left[\left|d^{\prime} \omega\right|^{2}+\left|\delta^{\prime} \omega\right|^{2}+\left|\delta^{\prime} \omega-\delta_{0}^{\prime} \omega\right|^{2}\right] .
\end{aligned}
$$

The proof will be completed if we can show that $\left|\delta^{\prime} \omega-\delta_{0}^{\prime} \omega\right|^{2} \leqq C_{2}|\omega|^{2}$ $+\epsilon / 2 C_{1} D_{0}^{\prime}(\omega)$. A component of $\delta^{\prime} \omega-\delta_{0}^{\prime} \omega$ has the form

$$
\begin{aligned}
& -\sum\left(g^{\prime h k}-\delta^{h k}\right) \partial \omega_{h i_{2} \cdots i_{r} j_{1} \cdots j_{s}} / \partial x^{k} \\
& +g_{i_{2} k_{2}} \cdots g_{i_{r} k_{r}} g^{-1 / 2}\left(\partial / \partial x^{k_{1}}\right)\left(g^{1 / 2} g^{k_{1} l_{1}} \cdots g^{k_{r} l_{r}}\right) \omega_{l_{1}} \cdots l_{r} j_{1} \cdots j_{s}
\end{aligned}
$$

the first term vanishes at the origin by our choice of coordinate system. Thus, the integral of such terms may be made small by choosing the neighborhood sufficiently small. The second term has bounded coefficients, so the part of 
the norm due to it may be made less than $C_{2}|\omega|^{2}$.

Lemma 6.2 follows from Lemma 6.1 by a proof of Morrey and Eells $[16$, p. 121]. Choose a finite covering by neighborhoods of the type described in Lemma 6.1, and a partition of unity $\sum \phi_{\alpha}$ subordinate thereto. If Lemma 6.2 were false, there would be a sequence $\left\{\omega_{\beta}\right\}$ of forms such that $D^{\prime}\left(\omega_{\beta}\right)$ and $\left|\omega_{\beta}\right|^{2}$ were uniformly bounded, but $\| \omega_{\beta}||^{\prime} \rightarrow \infty$. Similar statements hold for $\left\{\phi_{\alpha} \omega_{\beta}\right\}$ for some $\alpha$ and a subsequence of $\omega_{\beta}$. But this contradicts Lemma 6.1 .

We are now prepared to carry out the construction of the Green's operator. Let $\phi$ be a section of $E=\sum D_{x}^{r} \wedge D_{y}^{s}$, and let $B$ be a coherent sheaf having $\phi$ as a section. $d^{\prime} \phi$ is a section of $B$; so are $\delta^{\prime} \phi$ and $\Delta^{\prime} \phi$ by reason of the torsionless metric. We construct the Green's operator $G^{\prime}$ in $\mathscr{L}_{2}(B)$; later we show that a different choice of $B$ would lead to the same $G^{\prime} \phi$.

Lemma 6.3. Let $\mathfrak{T}$ be a subspace in $\mathfrak{L}_{2}(B)$. Then either there is no form of $\mathfrak{M}$ which is in $\mathcal{P}_{2}^{\prime}(B)$ or there exists $\omega_{0}$ in $\mathfrak{M} \cap \mathcal{P}_{2}^{\prime}(B)$ with $\left|\omega_{0}\right|^{2}=1$ and which minimizes $D^{\prime}(\omega)$ among all such forms.

Again we draw our proof from Morrey and Eells. Choose a sequence $\left\{\omega_{\beta}\right\}$ such that $\left|\omega_{\beta}\right|^{2}=1, \omega_{\beta} \in \mathfrak{M} \cap \mathcal{P}_{2}^{\prime}(B)$, and $D^{\prime}\left(\omega_{\beta}\right)$ approaches its infimum. By Lemma 6.2, $\left\|\omega_{\beta}\right\|^{\prime}$ are uniformly bounded. Choose a subsequence converging weakly in $\boldsymbol{P}_{2}^{\prime}(B)$ to some form $\omega_{0}$. By Proposition 6.3, $\omega_{\beta}$ converges strongly to $\omega_{0}$ in $\mathfrak{L}_{2}(B)$. Also, $D^{\prime}(\omega)$ is lower semi-continuous with respect to weak convergence in $\mathcal{P}_{2}^{\prime}(B)$. This concludes the proof.

Lemma 6.4. The subspace $H^{\prime}(B)$ consisting of forms in $\mathcal{P}_{2}^{\prime}(B)$ satisfying $d^{\prime} \omega=\delta^{\prime} \omega=0$ is finite dimensional.

Using Lemma 6.3, construct a sequence $\omega_{\beta}$ of forms in $\mathscr{L}_{2}(B)$ such that $\omega_{1}$ minimizes $D^{\prime}(\omega)$ over $\mathscr{L}_{2}(B) \cap \mathcal{P}_{2}^{\prime}(B), \omega_{2}$ minimizes $D^{\prime}(\omega)$ over $\left(\mathfrak{L}_{2}(B) \theta \omega_{1}\right)$ $\cap \rho_{2}^{\prime}(B)$, etc., where $\mathscr{L}_{2}(B) \theta \omega_{1}$ is the orthogonal complement of $\omega_{1}$ in $\mathscr{L}_{2}(B)$. If $D^{\prime}\left(\omega_{\beta}\right)$ were zero for all $\beta$, we would have an infinite orthonormal sequence of forms converging strongly in $\mathfrak{L}_{2}(B)$ to a form of norm 1 , which is impossible. For more details, see the paper of Morrey and Eells [16, p. 122].

Lemma 6.5. $D^{\prime}(\omega) \geqq \lambda_{0}\|\omega\|^{\prime}$ for any $\omega$ in $\odot_{2}^{\prime}(B)$ which is orthogonal to $H^{\prime}(B)$.

For the proof, see Morrey and Eells [16, p. 122].

We are now in a position to state a theorem which, when combined with a differentiability theorem, will give us the Green's operator.

TheORem 6.1. Let $\omega_{0}$ be a form in $\mathfrak{L}_{2}(B)$ orthogonal to $H^{\prime}(B)$. Then there exists a unique form $\Omega_{0}$ in $\mathcal{P}_{2}^{\prime}(B)$ and orthogonal to $H^{\prime}(B)$ such that

$$
\left(d^{\prime} \Omega_{0}, d^{\prime} \zeta\right)+\left(\delta^{\prime} \Omega_{0}, \delta^{\prime} \zeta\right)=\left(\omega_{0}, \zeta\right)
$$

for all $\zeta$ in $\mathcal{P}_{2}^{\prime}(B)$. Furthermore, the map from $\omega_{0}$ to $\Omega_{0}$ is a bounded linear transformation from $\mathfrak{L}_{2}(B)$ into $\mathcal{P}_{2}^{\prime}(B)$. 
The proof follows one of Morrey and Eells [16, pp. 122-123]. We define $I^{\prime}(\omega)=D^{\prime}(\omega)-2\left(\omega, \omega_{0}\right)$, and establish the existence of a minimizing form $\Omega_{0}$ for $I^{\prime}(\omega)$. Examination of the minimizing conditions leads to a proof of the theorem.

If $\Omega_{0}$ were sufficiently differentiable, we would have

$$
\left(d^{\prime} \Omega_{0}, d^{\prime} \zeta\right)+\left(\delta^{\prime} \Omega_{0}, \delta^{\prime} \zeta\right)=\left(\Delta^{\prime} \Omega_{0}, \zeta\right)=\left(\omega_{0}, \zeta\right)
$$

for all $\zeta$ in $\mathcal{P}_{2}^{\prime}(B)$, which is dense in $\mathscr{L}_{2}(B)$; hence $\Delta^{\prime} \Omega_{0}=\omega_{0}$. We then define $G_{B}^{\prime} \omega_{0}=\Omega_{0}$. We can complete the construction of the Green's operator in $\mathscr{L}_{2}(B)$ if we prove the following:

THEOREM 6.2. Let $V$ be a compact almost product manifold with torsionless metric, and let $B$ be a coherent subsheaf of $E$. If $\left(\Omega_{0}, \Delta^{\prime} \zeta\right)=\left(\omega_{0}, \zeta\right)$ for all $\zeta$ in $\mathfrak{L}_{2}(B)$ which are $C^{\infty}$, and if $\omega_{0}$ is in $\mathfrak{L}_{2}(B)$ and is $C^{\infty}$, then $\Omega_{0}$ is $C^{\infty}$.

We cannot prove this theorem outside $\mathscr{L}_{2}(B)$ without some restriction; for example, the forms in $\mathfrak{L}_{2}$ satisfying $\Delta^{\prime} \Omega=0$ may depend upon $y$ in an arbitrary $\mathscr{L}_{2}$ fashion, at least in the case of a product space.

Since the metric is torsionless, the measure induced on a local product coordinate neighborhood is a product measure. Hence, Fubini's theorem applies, so that we may integrate over the neighborhood by integrating with respect to $x$ and $y$ successively. Thus in the neighborhood of any point, we may choose a local basis $\left\{\gamma_{i}\right\}$ for $B$ such that it is orthonormal on the space of $y$ coordinates. Any form in $\mathfrak{L}_{2}(B)$ may then be shown to be $C^{\infty}$ if and only if in each such neighborhood $\phi=\sum_{i} \phi_{i}(x) \wedge \gamma_{i}(y)$ with each $\phi_{i}(x) C^{\infty}$. In fact, let $\phi$ be $C^{\infty}$; since $\gamma_{j}(y)$ is $C^{\infty}$, so is

$$
\sum_{(j)(k)} \delta_{j_{1} \cdots j_{s} k_{1} \cdots k_{q-s}}^{1 \cdots \gamma_{j} \cdots j^{\prime}}(y)\left(g^{\prime \prime}(y)\right)^{+1 / 2} d y^{k_{1}} \wedge \cdots \wedge d y^{k_{q-\bullet}} .
$$

Thus

$$
\sum_{(j)} \phi_{i i_{1} \cdots i_{r}}(x) \gamma_{i j_{1} \cdots j_{s}}(y) \gamma_{j}^{j_{1} \cdots j_{s}}(y)\left(g^{\prime \prime}(y)\right)^{+1 / 2} d x^{i_{1}} \wedge \cdots
$$

$$
\wedge d x^{i_{r}} \wedge d y^{1} \wedge \cdots \wedge d y^{q}
$$

and its integral over $y$ are also. By the orthonormality, this integral is $\phi_{i i_{1}} \cdots i_{r} d x^{i_{1}} \wedge \cdots \wedge d x^{i_{r}}$, which is therefore $C^{\infty}$.

These facts enable us to restrict our consideration to forms $\omega_{0}$ and $\zeta$ which have their support contained in some local product coordinate neighborhood, and such that they are expressible as a single term $\phi_{i} \wedge \gamma_{i}$. The proof of the theorem then follows the method of Kodaira [14, pp. 608-626]. We begin by choosing in this neighborhood orthonormal coordinates consistent with the local product structure; we now show that if the metric is torsionless, such coordinates exist. 
Let $\left(x^{1}, \cdots, x^{p}, y^{1}, \cdots, y^{q}\right)$ be local coordinates, and define

$$
X_{j}=\sum g_{j k}^{\prime}(x) d x^{k} / d t, \quad Y_{j}=\sum g_{j k}^{\prime \prime}(y) d y^{k} / d t
$$

where $t$ is a multiple of the arc length along a geodesic. Define the Hamiltonian $H(x, y, X, Y)=1 / 2 \sum_{i, k} g^{\prime i k}(x) X_{i} X_{k}+1 / 2 \sum_{i, k} g^{\prime \prime i k}(y) Y_{i} Y_{k}=H^{\prime}(x, X)$ $+H^{\prime \prime}(y, Y)$.

The equations for a geodesic are:

$$
\begin{aligned}
& d x^{i} / d t=\partial H^{\prime} / \partial H X_{i}, \quad d X_{i} / d t=-\partial H^{\prime} / \partial x^{i}, \quad i=1, \cdots, p \\
& d y^{i} / d t=\partial H^{\prime \prime} / \partial Y_{i}, \quad d Y_{i} / d t=-\partial H^{\prime \prime} / \partial y^{i}, \quad i=1, \cdots, q .
\end{aligned}
$$

Two of these equations involve only $x$, the other two only $y$. Hence, given a point $(\xi, \eta)$ and a velocity $(\Xi, \mathrm{H})$, there is one and only one solution of these equations satisfying $x^{i}(0 ; \zeta, \Xi)=\xi^{i}, X_{i}(0 ; \xi, \Xi)=\Xi_{i}, y^{i}(0 ; \eta, \mathrm{H})=\eta^{i}$, and $Y_{i}(0 ; \eta, \mathrm{H})=\mathrm{H}_{i}$. The solutions are $C^{\infty}$ in $t$ and the other variables, and define for fixed $(\xi, \eta)$ a system of normal coordinates $\left(\Xi^{1}, \cdots, \Xi^{p}, \mathrm{H}^{1}, \cdots, \mathrm{H}^{q}\right)$ in the neighborhood of $t=0$. Here $\Xi^{i}=g^{\prime i k} \mathrm{H}_{k}$, etc. In terms of the normal coordinates $(\Xi, \mathrm{H})$ of a point $p$ in a coordinate system with origin $q$, we have for the distance $r(p, q)$ the formula

$$
r(p, q)=\left(\sum g_{i k}^{\prime}(\zeta) \Xi^{i} \Xi^{k}+\sum g_{i k}^{\prime \prime}(\eta) \mathrm{H}^{i} \mathrm{H}^{k}\right)^{1 / 2} .
$$

Here $(\xi, \eta)$ are the coordinates at $q$. Now there exist $h_{j}^{\prime \nu}(\xi)$ of $C^{\infty}$ such that $g_{j k}^{\prime}(\xi)=\sum_{\nu=1}^{p} h_{j}^{\prime \nu}(\xi) h_{k}^{\prime \nu}(\xi)$ and similarly for $h_{j}^{\prime \prime \nu}(\eta)$. Setting $\phi^{\nu}=\sum_{j} h_{j}^{\prime \nu}(\xi) \Xi^{j}$ and $\psi^{\nu}=\sum_{j} h_{j}^{\prime \prime \nu} \mathrm{H}^{j}$, we find $r^{2}(p, q)=\left(\phi^{\nu}\right)^{2}+\left(\psi^{\nu}\right)^{2}$. Then $\left(\phi^{1}, \cdots, \phi^{p}\right.$, $\left.\psi^{1}, \cdots, \psi^{a}\right)$ form a set of local product coordinates known as orthonormal coordinates.

The existence of these coordinates and of the product measure justifies our working in the $x$ space alone to construct a parametrix $\gamma^{\prime}$. Let $U_{x} \times V_{y}$ be a local product coordinate neighborhood of the kind occurring in the definition of $B$, and let $U_{1 x}$ be contained in $U_{x}$ and such that the distance measured in $x$ space from $U_{1 x}$ to the boundary of $U_{x}$ is greater than $3 \rho$. Let $(x, \xi)$ be a point in $U_{x} \times U_{1 x}$. Then there exists a differential form $\gamma^{\prime}$ such that:

(i) $\gamma^{\prime}=\gamma_{i_{1} \cdots i_{r} k_{1} \cdots k_{r}}^{\prime}(x, \xi) d x^{i_{1}} \wedge \cdots \wedge d x^{i_{r}} \wedge d \xi^{k_{1}} \wedge \cdots \wedge d \xi^{k_{r}}$ is $C^{\infty}$ in $x$ and in $\xi$ except on the diagonal of $U_{x} \times U_{1 x}$. If $r^{\prime}(x, \xi)$ is the geodesic distance between $x$ and $\xi$ considered as points of $x$ space, then $\left[r^{\prime}(x, \xi)\right]^{p-2} \gamma^{\prime}(x, \xi)$ is $C^{\infty}$ for $x=\xi$ and $p>2$. If $p=2,\left[\log r^{\prime}(x, \xi)\right] \gamma^{\prime}(x, \xi)$ is $C^{\infty}$ for $x=\xi$.

(ii) For fixed $\xi$, the carrier of $\gamma^{\prime}(x, \xi)$ has diameter less than $\rho$ for some given $\rho>0$.

(iii) $\Delta_{x}^{\prime} \gamma^{\prime}(x, \zeta)=q^{\prime}(x, \xi)$ is a form of class $C^{\nu}$ in $x$ and in $\xi$. Here $\Delta_{x}^{\prime}$ is by definition ordinary $\Delta$ in $x$ space.

The construction may be made by the method of Kodaira [14, pp. 608626], which gives an elementary solution such that $\Delta_{x}^{\prime} \gamma^{\prime}(x, \xi)=0$. By cutting 
off the power series for $\gamma^{\prime}$ after a finite number of terms, convergence questions are avoided; by taking a sufficiently large number of terms, $q^{\prime}(x, \xi)$ may be made $C^{\nu}$ for some preassigned $\nu$. Using the parametrix, we may then prove: $\left.{ }^{5}\right)$

(1) If $\phi$ is a form in $\mathscr{L}_{2}(B)$ which is $C^{\infty}, \phi=\phi^{\prime}(x) \wedge \gamma(y)$, and the carrier $\mathfrak{S}(\phi)$ is contained in $U_{1 x} \times V_{y}$, then $\psi(x, y)=\int_{\xi} \phi(\xi, y) \wedge * \xi \gamma^{\prime}(x, \xi)$ is $C^{\infty}, \mathfrak{\complement}(\psi)$ is contained in $U_{x} \times V_{y}$, and $\Delta^{\prime} \psi(x, y)=\phi(x, y)+\int_{\xi} \phi(\xi, y) \wedge * \xi q^{\prime}(x, \xi)$.

(2) If $\eta$ is a form in $\mathfrak{L}_{2}(B)$ which is $C^{\infty}, \eta=\eta^{\prime}(x) \wedge \gamma(y)$, and $\mathfrak{\complement}(\eta)$ is contained in $U_{x} \times V_{y}$, then $\int_{x} \Delta^{\prime} \eta(x, y) \wedge{ }{ }_{x} \gamma^{\prime}(x, \xi)=\eta(\xi, y)+\int_{x} \eta(x, y) \wedge{ }_{x} q^{\prime}(x, \xi)$.

(3) For any $\alpha$ in $\mathfrak{L}_{2}(B)$ on $U_{1 x} \times U_{y}$ with $\alpha=\alpha^{\prime}(x) \wedge \gamma(y)$,

$$
\int_{\xi} \alpha(\xi, y) \wedge * \xi q^{\prime}(x, \xi)
$$

is $C^{\nu}$. In these equations, $*_{x}$ is defined as that operator such that the second integrand in (2) becomes

$$
\begin{aligned}
\eta_{i_{1}} \cdots i_{r} j_{1} \cdots j_{s}(x, y) q^{\prime i_{1} \cdots i_{r_{2}}{ }_{k_{1}} \cdots k_{r}}(x, \zeta)\left(g^{\prime}(x)\right)^{1 / 2} d x^{1} \wedge & \cdots \wedge d x^{p} \wedge d \xi^{k_{1}} \wedge \cdots \\
& \wedge d \xi^{k_{r}} \wedge d y^{j_{1}} \wedge \cdots \wedge d y^{j_{s}}
\end{aligned}
$$

while $* \xi$ is that operator such that the integrand in (3) becomes $\alpha_{i_{1} \cdots i_{r} j_{1} \cdots j_{s}}(\xi, y) q_{k_{1} \cdots k_{r}} i_{1} \cdots i_{r}(x, \xi)\left(g^{\prime}(\xi)\right)^{1 / 2} d \xi^{1} \wedge \cdots \wedge d \xi^{p} \wedge d x^{k_{1}} \wedge \cdots$

$$
\wedge d x^{k_{r}} \wedge d y^{j_{1}} \wedge \cdots \wedge d y^{j_{s}} \text {. }
$$

Thus ${ }^{*}{ }_{x}$ is $(-1)^{p s}$ times the ordinary $*$ computed in $x$ space by ignoring the other variables and differentials, while $* \xi$ is $(-1)^{p m+r}$ times the ordinary $*$ in $\xi$ space. Here $m=r+s$.

The analogous statements for $\Delta$ are unpublished results of Kodaira. To prove the differentiability statements, we use:

Lemma 6.6. Let $\mu\left(r^{\prime}\right)$ be a function of $r^{\prime}$ such that $\left|\mu\left(r^{\prime}\right)\right| \leqq c / r^{\prime p-1}$, c a constant, and $\mu\left(r^{\prime}\right)=0$ for $r^{\prime} \geqq 2 \rho$. Let $W(x, \xi)$ be a double form of $C^{\infty}$ for $\xi$ in $U_{1 x} \times V_{y}$ and $x$ in $U_{x} \times V_{y}$. Let $\phi(\xi, y)$ be in $\mathfrak{L}_{2}(B)$, be $C^{\infty}$, satisfy $\phi=\phi^{\prime}(x) \wedge \gamma(y)$ and have $\mathfrak{S}(\phi)$ contained in $U_{1 x} \times V_{y}$. Then

$$
\psi(x, y)=\int_{\xi} \mu\left(r^{\prime}(x, \xi)\right) \phi(\xi, y) \wedge * \xi W(x, \xi)
$$

is $C^{\infty}$ with $\subseteq(\psi)$ contained in $U_{x} \times V_{y}$.

Introduce orthonormal coordinates $\left(\theta^{1}, \cdots, \theta^{p}, y^{1}, \cdots, y^{q}\right)$ with center $x$. Writing the integral in terms of these coordinates, we bound it by an expression which is essentially $\int_{S(2 \epsilon)} r^{1-p} d \theta^{1} \cdots d \theta^{p}$, where $S(2 \epsilon)$ is a ball of

${ }^{(5)}$ Added in proof. A construction of the paramatrix by the method of Kodaira, as well as another exposition of Kodaira's proof of the differentiability theorem, may be found in [28]. 
radius $2 \epsilon$ in euclidean $p$ space. Since this integral converges absolutely and uniformly, we may interchange differentiation and integration, which proves the theorem.

The differentiability statement in (1) is an immediate consequence of this result, since $\gamma^{\prime}(x, \xi)$ has the desired form by construction (see Kodaira $[14$, p. 618]). For statement (3), we have

$$
\begin{array}{r}
\frac{1}{\epsilon}\left[\int_{\xi} \alpha(\xi, y) \wedge * \xi q^{\prime}\left(x^{1}+\epsilon, x^{2}, \cdots, x^{p}, \xi\right)-\int_{\xi} \alpha(\xi, y) \wedge * \xi q^{\prime}\left(x^{1}, x^{2}, \cdots, x^{p}, \xi\right)\right] \\
=\int_{\xi} \alpha(\xi, y) \wedge * \xi \frac{1}{\epsilon}\left[q^{\prime}\left(x^{1}+\epsilon, x^{2}, \cdots, x^{p}, \xi\right)-q^{\prime}\left(x^{1}, x^{2}, \cdots, x^{p}, \xi\right)\right] ;
\end{array}
$$

since the expression in brackets converges to $\partial q^{\prime}(x, \xi) / \partial x^{1}$ uniformly with respect to $\xi$, the integral has a first derivative with respect to $x^{1}$. Similarly we show that the integral has continuous derivatives with respect to $x$, and indeed up to the same order as the kernel. The remainder of statement (1) is an immediate consequence of (2). Indeed, supposing (2) holds, we have for $C^{\infty} \eta$

$$
\begin{aligned}
\left(\eta, \Delta^{\prime} \psi\right) & =\left(\Delta^{\prime} \eta, \psi\right)=\left(\Delta^{\prime} \eta, \int_{\xi} \phi(\xi, y) \wedge * \xi \gamma^{\prime}(x, \xi)\right) \\
& =\left(\phi, \int_{x} \Delta^{\prime} \eta(x, y) \wedge{ }^{*} \gamma^{\prime}(x, \xi)\right)=(\phi, \eta)+\left(\phi, \int_{x} \eta(x, y) \wedge{ }_{x} q^{\prime}(x, \xi)\right) \\
& =(\eta, \phi)+\left(\eta, \int_{\xi} \phi(\xi, y) \wedge * \xi q^{\prime}(x, \xi)\right)
\end{aligned}
$$

therefore (1) holds. Here we have used the interchange of order of an integration involving the singular kernel $\gamma^{\prime}$; however, since $\gamma^{\prime}$ is $O\left(r^{p-2}\right)$, the integrals converge absolutely and Fubini's theorem applies. Conversely, by a similar proof, statement (1) implies statement (2).

In order to complete the proof of (1) and (2), we reduce to the case of a 0 form. Notice that (1) may be written

$$
\phi(x, y)=\left(\Delta^{\prime} \int_{\xi}-\int_{\xi} \Delta_{x}^{\prime}\right) \phi(\xi, y) \wedge * \xi \gamma^{\prime}(x, \xi) .
$$

Referring to the explicit formula for $\Delta^{\prime}$ in the case of a torsionless metric, we find that $\Delta^{\prime}$ has the property of acting in the $x$ space independently of the other variables; hence, $\Delta^{\prime}$ and $\Delta_{x}^{\prime}$ operate in the same way. Let us write the components of $\gamma^{\prime}$ as a matrix $\left(\gamma_{(k)}^{\prime(i)}\right)$ with rows $(i)$ and columns $(k)$ and the components of $\phi$ as a matrix $\left(\phi_{(i)(j)}\right)$ with rows $(j)$ and columns $(i)$. Then our equation becomes

$$
\left(\phi_{(k)(j)}(x, y)\right)=\left(\Delta^{\prime} \int_{\xi}-\int_{\xi} \Delta^{\prime}\left(\left(\gamma^{\prime(i)}(x, \xi)\right)\left(\phi_{(i)(j)}(\xi, y)\right)\left(g^{\prime}(\xi)\right)^{1 / 2} d \xi^{1} \cdots d \xi^{p}\right.\right.
$$


as is easily checked by writing down the original integral in terms of the components of the forms. Furthermore, the finiteness of

$$
\int|\partial F(x, \xi) / \partial x| d x d \xi
$$

implies that differentiation and integration may be interchanged. Applying this to our matrix equation, we find it reduces to

$$
\begin{aligned}
& \left(\phi_{(k)(j)}(x, y)\right) \\
& \quad=-\sum g^{\prime i k}(x)\left(\frac{\partial^{2}}{\partial x^{i} \partial x^{k}} \int_{\xi}-\int_{\xi} \frac{\partial^{2}}{\partial x^{i} \partial x^{k}}\right)\left[r^{\prime}(x, \xi)\right]^{2-p}\left(\phi_{(k)(j)}(\xi, y)\right) d V,
\end{aligned}
$$

where $d V=\left(g^{\prime}(\xi)\right)^{1 / 2} d \xi^{1} \cdots d \xi^{p}$, for all other terms of $\Delta_{x}^{\prime} \gamma^{\prime}(x, \xi)$ are $O\left(r^{1-p}\right)$ or better, so that the integrals converge absolutely and the interchange of differentiation and integration is valid. Picking off the components of this matrix formula, we are reduced to the case of a function, as desired.

We now prove statement (2) for functions. First, since $s=0, *_{x}$ becomes the same as $*$ computed in $x$ space; we have already seen that $\Delta^{\prime}$ and $\Delta_{x}^{\prime}$ may be identified. For the remainder of the proof, we shall require that our operations be in $x$ space and be unaffected by the other variables present; we shall denote such operations by affixing ' to the symbols. Our theorem then says, for a scalar $\eta$,

$$
\begin{aligned}
\eta(\xi, y) & =\int_{x} \eta(x, y) \wedge *^{\prime} \Delta^{\prime} \gamma^{\prime}(x, \xi)-\int_{x} \Delta^{\prime} \eta(x, y) \wedge *^{\prime} \gamma^{\prime}(x, \xi) \\
& =-\int_{x} d^{\prime}{ }^{\prime}{ }^{\prime}\left(\gamma^{\prime} d^{\prime} \eta-\eta d^{\prime} \gamma^{\prime}\right) .
\end{aligned}
$$

To show that the last expression equals the given one, commute ${ }^{\prime}$ with the scalar, apply $d^{\prime}$ to each term, replace $d^{\prime} *^{\prime}$ by $-*^{\prime} \delta^{\prime}$, and use $\Delta^{\prime}=\delta^{\prime} d^{\prime}$ which holds for a scalar. Let $S_{\epsilon}$ be an $\epsilon$ sphere about $\xi, S$ be the boundary of $U_{x}$, and $0_{\boldsymbol{\epsilon}}$ the volume bounded by these surfaces. Then, by Stokes' theorem

$$
\begin{aligned}
\int d^{\prime} *^{\prime}\left(\gamma^{\prime} d^{\prime} \eta-\eta d^{\prime} \gamma^{\prime}\right) & =\lim _{\epsilon \rightarrow 0} \int_{0_{\epsilon}} d^{\prime} *^{\prime}\left(\gamma^{\prime} d^{\prime} \eta-\eta d^{\prime} \gamma^{\prime}\right) \\
& =-\lim _{\epsilon \rightarrow 0} \int_{S_{\epsilon}} *^{\prime}\left(\gamma^{\prime} d^{\prime} \eta-\eta d^{\prime} \gamma^{\prime}\right)=\lim _{\epsilon \rightarrow 0} \int_{S_{\epsilon}} *^{\prime} \eta d^{\prime} \gamma^{\prime}
\end{aligned}
$$

the last equality holding because the area of $S_{\epsilon}$ is $O\left(\epsilon^{p-1}\right)$ while $\gamma^{\prime}$ is $O\left(\epsilon^{2-p}\right)$ and $d^{\prime} \eta$ is bounded. This last expression equals

$$
\lim _{\epsilon \rightarrow 0} 1 /(p-2) \omega_{p} \int_{S_{\epsilon}} \eta *^{\prime} d^{\prime}\left[r^{\prime}(x, \xi)\right]^{2-p}
$$


because the other terms of $\gamma^{\prime}$ are $O\left(r^{\prime 3-p}\right)$. Here $\omega_{p}$ is the area of the unit $p$ sphere; the constant factor comes from the explicit expression for $\gamma^{\prime}$ (Kodaira $\left[14\right.$, p. 618]). Since $\eta$ is $C^{\infty},|\eta(x, y)-\eta(\xi, y)|=O(\epsilon)$ on $S_{\xi}$; this enables us to write for the integral

$$
\begin{aligned}
& \frac{\eta(\xi, y)}{(p-2) \omega_{p}} \lim _{\epsilon \rightarrow 0} \int_{S_{\epsilon}} *^{\prime} d^{\prime}\left[r^{\prime}(x, \xi)\right]^{2-p} \\
&=\frac{\eta(\xi, y)}{(p-2) \omega_{p}} \lim _{\epsilon \rightarrow 0}\left[-(p-2) / 2 \epsilon^{-p} \int_{S_{\epsilon}} *^{\prime} d^{\prime}\left[r^{\prime}(x, \xi)\right]^{2}\right] .
\end{aligned}
$$

Again applying Stokes' theorem and integrating over the interior of $S_{\boldsymbol{\epsilon}}$, making use of the explicit expression for $d^{\prime} *^{\prime} d^{\prime}\left[r^{\prime}(x, \xi)\right]^{2}=-*^{\prime} \Delta^{\prime}\left[r^{\prime}(x, \xi)\right]^{2}$, we get finally

$$
\int_{x} \eta(x, y) \wedge *^{\prime} \Delta^{\prime} \gamma^{\prime}(x, \xi)-\int_{x} \Delta^{\prime} \eta(x, y) \wedge *^{\prime} \gamma^{\prime}(x, \xi)=\eta(\xi, y)
$$

which completes the proof of the formulas for the parametrix.

We may now prove Theorem 6.2 as follows:

Let $\zeta$ be an arbitrary form in $\mathfrak{L}_{2}(B)$ of $C^{\infty}$ in $x$ with small carrier and let $\theta(x, y)=\int_{s} \zeta(\xi, y) \wedge{ }_{*} \gamma^{\prime}(x, \xi)$ so that $\theta(x, y)$ is also $C^{\infty}$ in $x$, and $\Delta^{\prime} \theta(x, y)$ $=\zeta(x, y)+\int_{\xi} \zeta(\xi, y) \wedge * \xi q^{\prime}(x, \xi)$. Then

(I) $\left(\omega_{0}, \theta\right)=\left(\Omega_{0}, \Delta^{\prime} \theta\right)=\left(\Omega_{0}, \zeta\right)+\left(\left(\Omega_{0}(x, y), \int_{\xi} \zeta(\xi, y) \wedge * \xi q^{\prime}(x, \xi)\right)\right.$,

(II) $\left(\omega_{0}, \theta\right)=\int_{x, y} \omega_{0}(x, y) \wedge * \theta(x, y)=\int_{x, y} \omega_{0}(x, y) \wedge *\left[\int_{\xi} \zeta(\xi, y) \wedge * \xi \gamma^{\prime}(x, \xi)\right]$

$$
\begin{aligned}
& =\int_{\xi, y} \zeta(\xi, y) \wedge *\left[\int_{x} \omega_{0}(x, y) \wedge *{ }_{x} \gamma^{\prime}(x, \xi)\right] \\
& =\left(\zeta(\xi, y), \int_{x} \omega_{0}(x, y) \wedge *{ }_{x} \nu^{\prime}(x, \xi)\right),
\end{aligned}
$$

(III) $\left(\Omega_{0}(x, y), \int_{\xi} \zeta(\xi, y) \wedge * \xi q^{\prime}(x, \xi)\right)$

$$
\begin{aligned}
& =\int_{x, y} \Omega_{0}(x, y) \wedge *\left[\int_{\xi} \zeta(\xi, y) \wedge * \xi q^{\prime}(x, \xi)\right] \\
& =\int_{\xi, y} \zeta(\xi, y) \wedge *\left[\int_{x} \Omega_{0}(x, y) \wedge *{ }_{x} q^{\prime}(x, \xi)\right] \\
& =\left(\zeta(\xi, y), \int_{x} \Omega_{0}(x, y) \wedge{ }_{x} q^{\prime}(x, \xi)\right) .
\end{aligned}
$$


Substituting (II) and (III) in (I), which holds for a dense set of forms $\zeta$, we find that as an equation in $\mathfrak{L}_{2}(B)$

$$
\Omega_{0}(\xi, y)=\int_{x} \omega_{0}(x, y) \wedge{ }_{*} \gamma^{\prime}(x, \xi)-\int_{x} \Omega_{0}(x, y) \wedge{ }_{x} q^{\prime}(x, \xi) ;
$$

the first integral is $C^{\infty}$ in $\xi$, while the second is $C^{v}$ in $\xi$. Both integrals being in $\mathfrak{L}_{2}(B)$, they are $C^{\infty}$ in $y$. As we have previously remarked, it is sufficient to consider the case that $\omega_{0}$ is locally the product of a form in $\xi$ with a form in $y$, and that $\Omega_{0}$ has the same property. It follows that each integral is $C^{\nu}$ in all variables together. Hence the equation holds pointwise, and $\Omega_{0}(x, y)$ is $C^{\nu}$ for every $\nu$, therefore $C^{\infty}$. This proves Theorem 6.2 .

Combining Theorems 6.1 and 6.2, we can construct the Green's operator. First let us close the operators $d^{\prime}$ and $\delta^{\prime}\left({ }^{4}\right)$. Then by a method of M. H. Stone (for which see Gaffney [9, pp. 434-435]) we can show that $\Delta^{\prime}=d^{\prime} \delta^{\prime}+\delta^{\prime} d^{\prime}$ is a self-adjoint operator, in particular is also closed. Now, applying Theorem 6.1, we know that given $\omega_{0}$ orthogonal to $H^{\prime}(B)$, we can find $\Omega_{0}$ orthogonal to $H^{\prime}(B)$ such that $\left(d^{\prime} \Omega_{0}, d^{\prime} \zeta\right)+\left(\delta^{\prime} \Omega_{0}, \delta^{\prime} \zeta\right)=\left(\omega_{0}, \zeta\right)$; if $\omega_{0}$ is $C^{\infty}$, then by Theorem $6.2 \Omega_{0}$ is $C^{\infty}$, whence $\Delta^{\prime} \Omega_{0}=\omega_{0}$. Define $G_{B}^{\prime}\left(\omega_{0}\right)=\Omega_{0}$; then $\Delta^{\prime} G_{B}^{\prime} \omega_{0}=\omega_{0}$. In particular, $H_{B}^{\prime} \phi$ is $C^{\infty}$ for any $\phi$, so that $\phi-H_{B}^{\prime} \phi$ is $C^{\infty}$ if $\phi$ is. Now let $\phi$ be an arbitrary form in $\mathscr{L}_{2}(B)$, and let $\left\{\phi_{\alpha}\right\}$ be a sequence of $C^{\infty}$ forms approximating $\phi$. Then $\left\{\omega_{\alpha}=\phi_{\alpha}-H_{B}^{\prime} \phi_{\alpha}\right\}$ is a sequence of $C^{\infty}$ forms approximating $\omega=\phi-H_{B}^{\prime} \phi$, and each $\omega_{\alpha}$ satisfies $\Delta^{\prime} G_{B}^{\prime} \omega_{\alpha}=\omega_{\alpha} \cdot G_{B}^{\prime}$ is a bounded operator by Theorem 6.1, since $\left|G_{B}^{\prime} \omega_{0}\right| \leqq\left\|G_{B}^{\prime} \omega_{0}\right\|^{\prime} \leqq K\left|\omega_{0}\right|$. Hence $G_{B}^{\prime} \omega_{\alpha} \rightarrow G_{B}^{\prime} \omega$, while $\Delta^{\prime} G_{B}^{\prime} \omega_{\alpha}=\omega_{\alpha} \rightarrow \omega$. Since $\Delta^{\prime}$ is a closed operator, we have $\Delta^{\prime} G_{B}^{\prime} \omega=\omega$. Let us define $G_{B}^{\prime} \phi=G_{B}^{\prime} \omega$. Then $\Delta^{\prime} G_{B}^{\prime} \phi=\phi-H_{B}^{\prime} \phi$. If $\phi$ is in $H^{\prime}(B)$, then $G_{B}^{\prime} \phi=0$; hence $G_{B}^{\prime} H_{B}^{\prime} \phi=0$. Also $H_{B}^{\prime} G_{B}^{\prime} \phi=0$ by Theorem 6.1. It follows immediately that $G_{B}^{\prime}$ is a symmetric operator on $\mathscr{L}_{2}(B)$, and $G_{B}^{\prime} \Delta^{\prime} \phi=\phi-H_{B}^{\prime} \phi$ for all $\phi$ in the domain of $\Delta^{\prime}$. We summarize these results in the following theorem:

THEOREM 6.3. There is defined on the Hilbert space $\mathcal{L}_{2}(B)$ a bounded symmetric operator $G_{B}^{\prime}$ such that $\Delta^{\prime} G_{B}^{\prime} \phi=G_{B}^{\prime} \Delta^{\prime} \phi=\phi-H_{B}^{\prime} \phi$ and $G_{B}^{\prime} H_{B}^{\prime} \phi=H_{B}^{\prime} G_{B}^{\prime} \phi$ $=0$.

Suppose now $B$ is a subsheaf of the coherent sheaf $C$, and let $\phi$ be in $\mathfrak{L}_{2}(B)$. Since $\phi$ is then also in $\mathfrak{L}_{2}(C)$, we may define $H^{\prime}{ }_{C} \phi$ and $G_{C}^{\prime} \phi$. We wish to show that $H_{C}^{\prime} \phi=H_{B}^{\prime} \phi$, from which it will follow that $G_{C}^{\prime} \phi=G_{B}^{\prime} \phi$. In fact, then the difference $G_{B}^{\prime} \phi-G_{C}^{\prime} \phi$ satisfies

$$
\begin{aligned}
& \Delta^{\prime}\left(G_{B}^{\prime} \phi-G_{C}^{\prime} \phi\right)=\phi-H_{B}^{\prime} \phi-\phi+H_{C}^{\prime} \phi=0, \\
& H_{C}^{\prime}\left(G_{B}^{\prime} \phi-G_{C}^{\prime} \phi\right)=H_{B}^{\prime} G_{B}^{\prime} \phi-H_{C}^{\prime} G_{C}^{\prime} \phi=0,
\end{aligned}
$$

so that $G_{B}^{\prime} \phi-G_{C}^{\prime} \phi=0$.

Now $\mathfrak{L}_{2}(B)$ is a subspace of $\mathfrak{L}_{2}(C)$, so that we may write an orthogonal decomposition $\mathfrak{L}_{2}(C)=\mathfrak{L}_{2}(B) \oplus \mathcal{D}$. For $\phi$ in $\mathfrak{L}_{2}(C)$, we have $\phi=\beta+\alpha$. Since 
$\Delta^{\prime}$ is a self-adjoint operator on $\mathscr{L}_{2}(B)$ and on $\aleph_{2}(C), \phi$ in the domain of $\Delta^{\prime}$ implies that $\beta$ and hence $\alpha$ are also; this in turn implies that $\Delta^{\prime}$ is a selfadjoint operator on $\mathcal{D}$. In particular, if $\Delta^{\prime} \phi=0$, then $\Delta^{\prime} \beta=\Delta^{\prime} \alpha=0$; hence, we may choose a base of $H^{\prime}(C)$ by choosing one of $H^{\prime}(B)$ and adjoining certain forms orthogonal to $H^{\prime}(B)$. These base forms define a kernel for the projection operator $H_{C}^{\prime}$. If we let this kernel operate on a form in $\mathfrak{L}_{2}(B)$, it is clear that its action is the same as that of the kernel for $H_{B}^{\prime}$; hence $H_{B}^{\prime} \phi=H_{C}^{\prime} \phi$. This completes the proof that $G_{B}^{\prime} \phi$ is independent of the choice of coherent subsheaf $B$ of $E$ containing $\phi$; for if $B$ and $C$ are any two such sheaves, we may find another such $D$ having $B$ and $C$ as subsheaves. Our final theorem may then be stated as follows:

THEOREM 6.4. Let $V$ be a compact almost product manifold with torsionless metric. Let $\phi$ be a $C^{\infty}$ section of the sheaf $E=\sum_{r, s} D_{s}^{r} \wedge D_{y}^{s}$. Then there is a $C^{\infty}$ section $G^{\prime} \phi$ of $E$ such that $\Delta^{\prime} G^{\prime} \phi=G^{\prime} \Delta^{\prime} \phi=\phi-H^{\prime} \phi$ and $G^{\prime} H^{\prime} \phi=H^{\prime} G^{\prime} \phi=0$. In these equations, $G^{\prime} \phi=G_{B}^{\prime} \phi$ and $H^{\prime} \phi=H_{B}^{\prime} \phi$ by definition, where $B$ is any coherent subsheaf of $E$ having $\phi$ as a section. $G^{\prime}$ commutes with $d^{\prime}$ and $\delta^{\prime} . G^{\prime}$ is not in general a bounded operator on the Hilbert space of all square integrable forms on the manifold.

We have proved all but the last two sentences. The commutation follows from an argument analogous to the proof of Proposition 5.3, by noticing that $d^{\prime}$ and $\delta^{\prime}$ commute with $\Delta^{\prime}$. If $G^{\prime}$ were bounded and densely defined, it would be everywhere defined. However, we saw in $\$ 4$ that $G^{\prime}$ may be densely defined without being everywhere defined; hence it must in general be unbounded. It is instructive to compare Theorem 6.4 more closely with the example of $\S 4$; in that example, $G^{\prime}$ is defined for all $C^{\infty}$ functions whose Fourier series have a finite number of terms. Such functions are sections of the sheaf $E$.

THEOREM 6.5. Let $V$ be a compact almost product manifold with torsionless metric. Then the $d^{\prime}$ cohomology of sections of the sheaf $E$ is isomorphic to the space of $\Delta^{\prime}$ harmonic sections of $E$, the isomorphism being given by assigning to each cohomology class the unique harmonic form contained in it.

In fact, Theorem 4 gives rise to decomposition $\phi=d^{\prime} \delta^{\prime} G^{\prime} \phi+\delta^{\prime} d^{\prime} G^{\prime} \phi+H^{\prime} \phi$ which together with the equation $d^{\prime} G^{\prime}=G^{\prime} d^{\prime}$ implies the theorem.

Theorem 6.5 implies that $E_{2}$ in a certain spectral sequence is isomorphic to $E_{\infty}$. We define this spectral sequence as follows: Let $A^{r, s}$ be the set of $C^{\infty}$ sections of the sheaf $E$ of type $(r, s)$ and let the filtration module $F^{p} A$ $=\sum_{r \geqq p, s \geq 0} A^{r, s}$. Then $E_{1}$ is the $d^{\prime \prime}$ cohomology of $C^{\infty}$ sections of $E$; this is isomorphic to the set of $\Delta^{\prime \prime}$ harmonic sections of $E$. $E_{2}$ is then isomorphic to the quotient of these elements of $H^{\prime \prime}$ satisfying $d^{\prime} H^{\prime \prime} \phi=d^{\prime \prime} \psi$ by these of the form $d^{\prime} \psi+d^{\prime \prime} \eta$. Using the decomposition given by Theorem 6.4 , we get

$$
H^{\prime \prime} \phi=d^{\prime} \delta^{\prime} G^{\prime} H^{\prime \prime} \phi+\delta^{\prime} d^{\prime} G^{\prime} H^{\prime \prime} \phi+H^{\prime} H^{\prime \prime} \phi ;
$$


using commutation of $d^{\prime}$ with $G^{\prime}$ and inserting the fact that $d^{\prime} H^{\prime \prime} \phi=d^{\prime \prime} \psi$, we get

$$
H^{\prime \prime} \phi=d^{\prime} \delta^{\prime} G^{\prime} H^{\prime \prime} \phi+\delta^{\prime} G^{\prime} d^{\prime \prime} \psi+H^{\prime} H^{\prime \prime} \phi .
$$

By torsionlessness, $\delta^{\prime} d^{\prime \prime}+d^{\prime \prime} \delta^{\prime}=0$, while by integrability, $d^{\prime} d^{\prime \prime}+d^{\prime \prime} d^{\prime}=0$; consequently, $d^{\prime \prime} \Delta^{\prime}=\Delta^{\prime} d^{\prime \prime}$. Let $\eta=d^{\prime \prime} H^{\prime} \phi-H^{\prime} d^{\prime \prime} \phi$; applying $\Delta^{\prime}$ to this equation, we see that $\eta$ is in $H^{\prime}$. On the other hand,

$$
\Delta^{\prime}\left(G^{\prime} d^{\prime \prime} \phi-d^{\prime \prime} G^{\prime} \phi\right)=\Delta^{\prime} \theta=\eta ; \text { thus }(\eta, \eta)=\left(\Delta^{\prime} \theta, \eta\right)=\left(\theta, \Delta^{\prime} \eta\right)=0 .
$$

Thus the following commutation relations hold: $d^{\prime \prime} \Delta^{\prime}=\Delta^{\prime} d^{\prime \prime}, d^{\prime \prime} H^{\prime}=H^{\prime} d^{\prime \prime}$, $d^{\prime \prime} G^{\prime}=G^{\prime} d^{\prime \prime}$, and $d^{\prime \prime} \delta^{\prime}=-\delta^{\prime} d^{\prime \prime}$. Using these in the decomposition of $H^{\prime \prime} \phi$, we find

$$
H^{\prime \prime} \phi=d^{\prime} \delta^{\prime} G^{\prime} H^{\prime \prime} \phi-d^{\prime \prime} \delta^{\prime} G^{\prime} \psi+H^{\prime} H^{\prime \prime} \phi .
$$

Since $H^{\prime} H^{\prime \prime} \phi$ cannot be cohomologous to $H^{\prime} H^{\prime \prime} \psi$ unless they are equal, each class in $E_{2}$ is represented by a unique form, which is easily proved to be harmonic in the usual sense. Thus $\operatorname{dim} \sum_{r+s=p} E_{2}^{r, s} \leqq b_{p}$, the $p$ th Betti number. On the other hand, by the nature of the spectral sequence, $\operatorname{dim} E_{2}^{r, s}$ $\geqq \operatorname{dim} E_{\infty}^{r, s}$; therefore, $\operatorname{dim} \sum_{r+s=p} E_{2}^{r, s}=b_{p}$ and $E_{2}$ is isomorphic to $E_{\infty}$. If $G^{\prime}$ and $G^{\prime \prime}$ are defined on all $C^{\infty}$ forms, the same theorem may be proved for the spectral sequence obtained by filtering all $C^{\infty}$ differential forms.

-REMARK. The ordinary construction fails to give a partition of unity for the sheaf $E$. In fact, for a covering $\mathfrak{U}=\left\{U_{\alpha}\right\}$ one normally chooses forms $\phi_{\alpha}$ satisfying $0 \leqq \phi_{\alpha} \leqq 1$ with $\mathfrak{S}\left(\phi_{\alpha}\right) \subset U_{\alpha}$, then divides by $\sum \phi_{\alpha}$ which is constructed to be nonzero at every point. In our case, we may choose $\phi_{\alpha}(x, y)$ $=\psi_{\alpha}(x) \mathrm{X}_{\alpha}(y)$; however, dividing by $\sum \phi_{\alpha}$ destroys the property of being sections of $E$. Thus, we are prevented from patching together local sections of $E$ to form global sections. Failing this, we cannot prove that $E$ is a fine sheaf, so cannot apply the standard sheaf theoretic techniques for proving a de Rham type of theorem for $d^{\prime}$ on $E$.

Finally, we shall consider various Hilbert space properties of $\Delta^{\prime}$ on a compact manifold. Many of the results we quote can be proved by methods of Gaffney ([8] and [9]) and Duff and Spencer [7]; the particular formulation we use is due to Conner [5]. Further references may be found in Conner's paper.

Recall that $d^{\prime}$ and $\delta^{\prime}$ are defined for all $C^{\infty}$ forms on $V$, and satisfy the equations $\left(d^{\prime} \phi, \psi\right)=\left(\phi, \delta^{\prime} \psi\right)$ and $d^{\prime 2}=\delta^{\prime 2}=0$. These facts enable us to define the closures of $d^{\prime}$ and $\delta^{\prime}$ in such a way that $d^{\prime}$ is the adjoint of $\delta^{\prime}$ and conversely. Henceforth in this section, $d^{\prime}$ and $\delta^{\prime}$ shall mean the closed operators. Proceeding in the manner of Conner [5], we show that the following are true of $\Delta^{\prime}$ :

(1) $\Delta^{\prime}$ is a positive self-adjoint operator.

(2) If $\mathcal{G}^{\prime}$ is the domain of $\Delta^{\prime}$, there exists an operator $G_{s}^{\prime}$ mapping $\mathscr{L}_{2}$ into $\mathcal{G}^{\prime}$ such that $\left(\Delta^{\prime}+s\right) G_{s}^{\prime}=I$ and $\left|G_{s}^{\prime}\right| \leqq 1 / s$. Here $s>0$. Furthermore, if $d^{\prime} \phi$ is 
defined, then $d^{\prime} G_{s}^{\prime} \phi=G_{s}^{\prime} d^{\prime} \phi . G_{s}^{\prime}$ may be obtained either from the theory of strictly positive transformations in Hilbert space, or from minimizing a Dirichlet integral.

(3) The eigenforms of $G_{s}^{\prime}$ for the eigenvalue $1 / s$ are the elements of the space $H^{\prime}$ of solutions of $d^{\prime} \phi=\delta^{\prime} \phi=0$.

(4) There is defined a semi-group $U_{t}^{\prime}$ such that:

(a) $U_{t}^{\prime}\left(U_{s}^{\prime}\right)=U_{t+s}^{\prime}$.

(b) $\partial U_{t}^{\prime} / \partial t=-\Delta^{\prime} U_{t}^{\prime}$ for $t>0$.

(c) $\lim _{t \rightarrow 0} U_{t}^{\prime}(\phi)=\phi$.

(d) $\lim _{t \rightarrow \infty} U_{t}^{\prime}(\phi)=H^{\prime} \phi$, where $H^{\prime} \phi$ is the projection of $\phi$ on the space $H^{\prime}$.

(e) $d^{\prime} U_{t}^{\prime}(\phi)=U_{t}^{\prime}\left(d^{\prime} \phi\right)$.

(5) If $\phi$ is a form such that $d^{\prime} \phi=0$, and if $\theta$ is such that $\delta^{\prime} \theta=0$, then $(\phi, \theta)=\left(H^{\prime} \phi, \theta\right)$.

In the theory of the operator $\Delta$, it is shown that any finite cycle $Z$ is representable as a form in the sense that $\int_{Z} \phi=(\phi, \theta)$ for any closed form $\phi$. A proof of this result is given by Kodaira [14, pp. 589-602]. The form $\theta$ satisfies $\delta \theta=0$; thus, the analog of statement (5) implies that given any closed form $\phi$, there is a harmonic form having the same periods on any finite cycle. The author does not know whether an analogous theory can be constructed for an appropriate kind of cycle on an almost product manifold. If it could, we should have a relation between the periods of a $d^{\prime}$ closed form and its $\Delta^{\prime}$ harmonic part.

Let us consider the map from the $d^{\prime}$ closed forms into $\Delta^{\prime}$ harmonic forms defined by the projection operator $H^{\prime}$. The projection is well-defined since the kernel of a closed operator is a subspace. Since $d^{\prime}$ is mapped into zero by this map, there is induced a homomorphism of the $d^{\prime}$ cohomology into $H^{\prime}$. This homomorphism is onto, for any form $\phi$ in $H^{\prime}$ belongs to some cohomology class, and the class of $\phi$ is mapped onto $\phi$ by the mapping considered. Our discussion of the torus has shown that the map is not in general an isomorphism. However, if the Green's operator is defined everywhere on $\mathfrak{L}_{2}$, we do obtain an isomorphism, by the argument of Theorem 6.5. Note that here we are discussing the cohomology of all square integrable forms, and not that of the sections of $E$.

\section{BibLIOGRAPHY}

1. A. Borel and A. Lichnerowicz, Groupes d'holonomie des variétés riemanniennes, C. R. Acad. Sci. Paris vol. 234 (1952) pp. 1835-1837. $1-42$.

2. E. Cartan, Les groupes d'holonomie des espaces génêralisés, Acta Math. vol. 48 (1926) pp.

3. S. S. Chern, Differentiable manifolds (Mimeographed notes), University of Chicago, 1950.

4. - Topics in differential geometry (Mimeographed notes), Princeton, Institute for Advanced Study, 1951.

5. P. E. Conner, The Neumann's problem for differential forms on Riemannian manifolds, Memoirs Amer. Math. Soc., no. 20, 1956. 
6. A. Denjoy, Sur les courbes définis par les équations différentielles à la surface du tore, J. Math. Pures Appl. ser. 9 vol. 11 (1932) pp. 333-375.

7. G. F. D. Duff and D. C. Spencer, Harmonic tensors on Riemannian manifolds with boundary, Ann. of Math. vol. 56 (1952) pp. 128-156.

8. M. P. Gaffney, The heat equation method of Milgram and Rosenbloom for open Riemannian manifolds, Ann. of Math. vol. 60 (1954) pp. 458-466.

9. - Hilbert space methods in the theory of harmonic integrals, Trans. Amer. Math. Soc. vol. 78 (1955) pp. 426-444.

10. V. K. A. M. Guggenheim and D. C. Spencer, Chain homotopy and the de Rham theory, Proc. Amer. Math. Soc. vol. 7 (1956) pp. 144-152.

11. A. Haefliger, Sur les feuilletages analytiques, C. R. Acad. Sci. Paris vol. 242 (1956) pp. 2908-2910.

12. E. R. van Kampen, The topological transformations of a simple closed curve into itself, Amer. J. Math. vol. 57 (1935) pp. 142-152.

13. H. Kneser, Reguläre Kurvenscharen auf den Ringflächen, Math. Ann. vol. 91 (1924) pp. $135-154$.

14. K. Kodaira, Harmonic fields in Riemannian manifolds (generalized potential theory), Ann. of Math. vol. 50 (1949) pp. 587-665.

15. J. F. Koksma, Diophantische Approximationen, Ergebnisse der Mathematik vol. 4 (1936) no. 4.

16. C. B. Morrey and J. Eells, A variational method in the theory of harmonic integrals, Ann. of Math. vol. 63 (1956) pp. 91-128.

17. H. K. Nickerson and D. C. Spencer, Differentiable manifolds and sheaves (Notes), Princeton University, 1955.

18. G. Reeb, Sur certaines propriétés topologiques des variêtés feuilletées, Paris, Hermann et Cie., 1952.

19. G. de Rham, Variétés différentiables, Paris, Hermann et Cie., 1955.

20. - - Sur la réductibilité d'un espace de Riemann, Comment. Math. Helv. vol. 26 (1952) pp. 328-344.

21. F. Riesz and B. Sz.-Nagy, Lȩ̧ons d'analyse fonctionnelle, 2d. ed., Budapest, Académie des Sciences de Hongrie, 1953.

22. M. Schiffer and D. C. Spencer, Functionals on finite Riemann surfaces, Princeton University Press, 1954.

23. Séminaire de topologie algébrique 1950-1951 (Polycopied notes), Paris, Secretariat Mathematique.

24. J.-P. Serre, Faisceaux algébriques cohérent, Ann. of Math. vol. 61 (1955) pp. 197-278.

25. C. L. Siegel, Note on differential equations on the torus, Ann. of Math. vol. 46 (1945) pp. $423-428$.

26. D. C. Spencer, Potential theory and almost complex manifolds, Lectures on Functions of a Complex Variable, Ann Arbor, University of Michigan Press, 1955.

27. N. E. Steenrod, The topology of fibre bundles, Princeton University Press, 1951.

28. Added in proof. W. L. Baily, Jr., The decomposition theory for V-manifolds, Amer. J. Math. vol. 78 (1956) pp. 862-888.

Princeton University, Princeton, New Jersey 